\title{
Nanosecond-level time synchronization of autonomous radio detector stations for extensive air showers
}

The Pierre Auger Collaboration

A. Aab ${ }^{41}$, P. Abreu ${ }^{67}$, M. Aglietta ${ }^{52,53}$, E.J. Ahn ${ }^{82}$, I. Al Samarai ${ }^{28}$, I.F.M. Albuquerque ${ }^{16}$, I. Allekotte ${ }^{1}$, P. Allison ${ }^{87}$, A. Almela ${ }^{11,8}$, J. Alvarez Castillo ${ }^{60}$, J. Alvarez-Muñiz ${ }^{77}$, R. Alves Batista ${ }^{40}$, M. Ambrosio ${ }^{43}$, A. Aminaei ${ }^{61}$, G.A. Anastasi ${ }^{45}$, L. Anchordoqui ${ }^{81}$, S. Andringa ${ }^{67}$, C. Aramo ${ }^{43}$, F. Arqueros ${ }^{74}$, N. Arsene ${ }^{70}$, H. Asorey ${ }^{1,24}$, P. Assis ${ }^{67}$, J. Aublin ${ }^{30}$, G. Avila ${ }^{10}$, N. Awal ${ }^{85}$, A.M. Badescu ${ }^{71}$, C. Baus ${ }^{35}$, J.J. Beatty ${ }^{87}$, K.H. Becker ${ }^{34}$, J.A. Bellido ${ }^{12}$, C. Berat ${ }^{31}$, M.E. Bertaina ${ }^{53,54}$, X. Bertou ${ }^{1}$, P.L. Biermann ${ }^{38}$, P. Billoir ${ }^{30}$, S.G. Blaess ${ }^{12}$, A. Blanco ${ }^{67}$, M. Blanco ${ }^{30}$, J. Blazek ${ }^{26}$, C. Bleve ${ }^{47}$, H. Blümer ${ }^{35,36}$, M. Boháčová ${ }^{26}$, D. Boncioli ${ }^{51}$, C. Bonifazi ${ }^{22}$, N. Borodai ${ }^{65}$, J. Brack ${ }^{80}$, I. Brancus ${ }^{68}$, T. Bretz ${ }^{39}$, A. Bridgeman ${ }^{36}$, P. Brogueira ${ }^{67}$, P. Buchholz ${ }^{41}$, A. Bueno ${ }^{76}$, S. Buitink ${ }^{61}$, M. Buscemi ${ }^{43}$, K.S. Caballero-Mora ${ }^{58}$, B. Caccianiga ${ }^{42}$, L. Caccianiga ${ }^{30}$, M. Candusso ${ }^{44}$, L. Caramete ${ }^{69}$, R. Caruso ${ }^{45}$, A. Castellina ${ }^{52,53}$, G. Cataldi ${ }^{47}$, L. Cazon ${ }^{67}$, R. Cester $^{46}$, A.G. Chavez ${ }^{59}$, A. Chiavassa ${ }^{53,54}$, J.A. Chinellato ${ }^{17}$, J. Chudoba ${ }^{26}$, M. Cilmo ${ }^{43}$, R.W. Clay ${ }^{12}$, G. Cocciolo ${ }^{47}$, R. Colalillo ${ }^{43}$, A. Coleman ${ }^{88}$, L. Collica ${ }^{53}$, M.R. Coluccia ${ }^{47}$, R. Conceição ${ }^{67}$, F. Contreras ${ }^{9}$, M.J. Cooper ${ }^{12}$, A. Cordier $^{29}$, S. Coutu ${ }^{88}$, C.E. Covault ${ }^{78}$, J. Cronin ${ }^{89}$, R. Dallier ${ }^{33,32}$, B. Daniel ${ }^{17}$, S. Dasso ${ }^{5,3}$, K. Daumiller ${ }^{36}$, B.R. Dawson ${ }^{12}$, R.M. de Almeida ${ }^{23}$, S.J. de Jong ${ }^{61,63}$, G. De Mauro ${ }^{61}$, J.R.T. de Mello Neto $^{22}$, I. De Mitri ${ }^{47}$, J. de Oliveira ${ }^{23}$, V. de Souza ${ }^{15}$, L. del Peral ${ }^{75}$, O. Deligny ${ }^{28}$, N. Dhital ${ }^{84}$, C. Di Giulio ${ }^{44}$, A. Di Matteo ${ }^{48}$, J.C. Diaz ${ }^{84}$, M.L. Díaz Castro ${ }^{17}$, F. Diogo ${ }^{67}$, C. Dobrigkeit ${ }^{17}$, W. Docters ${ }^{62}$, J.C. D'Olivo ${ }^{60}$, A. Dorofeev $^{80}$, Q. Dorosti Hasankiadeh $^{36}$, R.C. dos Anjos ${ }^{15}$, M.T. Dova ${ }^{4}$, J. Ebr $^{26}$, R. Engel ${ }^{36}$, M. Erdmann $^{39}$, M. Erfani ${ }^{41}$, C.O. Escobar ${ }^{82,17}$, J. Eser ${ }^{35}$, J. Espadanal ${ }^{67}$, A. Etchegoyen ${ }^{8,11}$, H. Falcke ${ }^{61,64,63}$, K. Fang ${ }^{89}$, G. Farrar ${ }^{85}$, A.C. Fauth ${ }^{17}$, N. Fazzini ${ }^{82}$, A.P. Ferguson ${ }^{78}$, B. Fick ${ }^{84}$, J.M. Figueira ${ }^{8}$, A. Filevich ${ }^{8}$, A. Filipčič ${ }^{72,73}$, O. Fratu ${ }^{71}$, M.M. Freire ${ }^{6}$, T. Fujii ${ }^{89}$, B. García ${ }^{7}$, D. García-Gámez ${ }^{29}$, D. Garcia-Pinto ${ }^{74}$, F. Gate ${ }^{33}$, H. Gemmeke ${ }^{37}$, A. Gherghel-Lascu ${ }^{68}$, P.L. Ghia ${ }^{30}$, U. Giaccari ${ }^{22}$, M. Giammarchi ${ }^{42}$, M. Giller ${ }^{66}$, D. Głas ${ }^{66}$, C. Glaser $^{39}$, H. Glass ${ }^{82}$, G. Golup ${ }^{1}$, M. Gómez Berisso ${ }^{1}$, P.F. Gómez Vitale $^{10}$, N. González ${ }^{8}$, B. Gookin ${ }^{80}$, J. Gordon ${ }^{87}$, A. Gorgi ${ }^{52,53}$, P. Gorham ${ }^{90}$, P. Gouffon ${ }^{16}$, N. Griffith ${ }^{87}$, A.F. Grillo ${ }^{51}$, T.D. Grubb ${ }^{12}$, F. Guarino ${ }^{43}$, G.P. Guedes ${ }^{18}$, M.R. Hampel ${ }^{8}$, P. Hansen ${ }^{4}$, D. Harari ${ }^{1}$, T.A. Harrison ${ }^{12}$, S. Hartmann ${ }^{39}$, J.L. Harton ${ }^{80}$, A. Haungs ${ }^{36}$, T. Hebbeker ${ }^{39}$, D. Heck ${ }^{36}$, P. Heimann ${ }^{41}$, A.E. Hervé ${ }^{36}$, G.C. Hill ${ }^{12}$, 
C. Hojvat ${ }^{82}$, N. Hollon ${ }^{89}$, E. Holt ${ }^{36}$, P. Homola ${ }^{34}$, J.R. Hörandel ${ }^{61,63}$, P. Horvath ${ }^{27}$, M. Hrabovský ${ }^{27,26}$, D. Huber ${ }^{35}$, T. Huege ${ }^{36}$, A. Insolia ${ }^{45}$, P.G. Isar ${ }^{69}$, I. Jandt ${ }^{34}$, S. Jansen ${ }^{61,63}$, C. Jarne ${ }^{4}$, J.A. Johnsen ${ }^{79}$, M. Josebachuili ${ }^{8}$, A. Kääpä ${ }^{34}$, O. Kambeitz ${ }^{35}$, K.H. Kampert ${ }^{34}$, P. Kasper ${ }^{82}$, I. Katkov ${ }^{35}$, B. Keilhauer ${ }^{36}$, E. Kemp ${ }^{17}$, R.M. Kieckhafer ${ }^{84}$, H.O. Klages ${ }^{36}$, M. Kleifges ${ }^{37}$, J. Kleinfeller ${ }^{9}$, R. Krause ${ }^{39}$, N. Krohm ${ }^{34}$, D. Kuempel ${ }^{39}$, G. Kukec Mezek ${ }^{73}$, N. Kunka ${ }^{37}$, A.W. Kuotb Awad ${ }^{36}$, D. LaHurd ${ }^{78}$, A. Lang ${ }^{35}$, L. Latronico ${ }^{53}$, R. Lauer ${ }^{92}$, M. Lauscher ${ }^{39}$, P. Lautridou ${ }^{33}$, S. Le $\mathrm{Coz}^{31}$, D. Lebrun ${ }^{31}$, P. Lebrun ${ }^{82}$, M.A. Leigui de Oliveira ${ }^{21}$, A. Letessier-Selvon ${ }^{30}$, I. Lhenry-Yvon ${ }^{28}$, K. Link ${ }^{35}$, L. Lopes ${ }^{67}$, R. López ${ }^{55}$, A. López Casado $^{77}$, K. Louedec ${ }^{31}$, A. Lucero ${ }^{8}$, M. Malacari ${ }^{12}$, M. Mallamaci ${ }^{42}$, J. Maller ${ }^{33}$, D. Mandat ${ }^{26}$, P. Mantsch ${ }^{82}$, A.G. Mariazzi ${ }^{4}$, V. Marin ${ }^{33}$, I.C. Mariş ${ }^{76}$, G. Marsella ${ }^{47}$, D. Martello ${ }^{47}$, H. Martinez ${ }^{56}$, O. Martínez Bravo ${ }^{55}$, D. Martraire ${ }^{28}$, J.J. Masías Meza ${ }^{3}$, H.J. Mathes ${ }^{36}$, S. Mathys ${ }^{34}$, J. Matthews ${ }^{83}$, J.A.J. Matthews ${ }^{92}$, G. Matthiae ${ }^{44}$, D. Maurizio ${ }^{13}$, E. Mayotte ${ }^{79}$, P.O. Mazur ${ }^{82}$, C. Medina ${ }^{79}$, G. Medina-Tanco ${ }^{60}$, R. Meissner ${ }^{39}$, V.B.B. Mello ${ }^{22}$, D. Melo ${ }^{8}$, A. Menshikov ${ }^{37}$, S. Messina ${ }^{62}$, M.I. Micheletti ${ }^{6}$, L. Middendorf ${ }^{39}$, I.A. Minaya ${ }^{74}$, L. Miramonti ${ }^{42}$, B. Mitrica ${ }^{68}$, L. Molina-Bueno ${ }^{76}$, S. Mollerach ${ }^{1}$, F. Montanet ${ }^{31}$, C. Morello ${ }^{52,53}$, M. Mostafá ${ }^{88}$, C.A. Moura ${ }^{21}$, G. Müller ${ }^{39}$, M.A. Muller ${ }^{17,20}$, S. Müller $^{36}$, S. Navas ${ }^{76}$, P. Necesal ${ }^{26}$, L. Nellen ${ }^{60}$, A. Nelles ${ }^{61,63}$, J. Neuser ${ }^{34}$, P.H. Nguyen ${ }^{12}$, M. Niculescu-Oglinzanu ${ }^{68}$, M. Niechciol ${ }^{41}$, L. Niemietz ${ }^{34}$, T. Niggemann ${ }^{39}$, D. Nitz ${ }^{84}$, D. Nosek ${ }^{25}$, V. Novotny ${ }^{25}$, L. Nožka ${ }^{27}$, L.A. Núñez ${ }^{24}$, L. Ochilo ${ }^{41}$, F. Oikonomou ${ }^{88}$, A. Olinto ${ }^{89}$, N. Pacheco ${ }^{75}$, D. Pakk Selmi-Dei ${ }^{17}$, M. Palatka ${ }^{26}$, J. Pallotta ${ }^{2}$, P. Papenbreer ${ }^{34}$, G. Parente ${ }^{77}$, A. Parra ${ }^{55}$, T. Paul ${ }^{81,86}$, M. Pech ${ }^{26}$, J. Pejkala ${ }^{65}$, R. Pelayo ${ }^{57}$, I.M. Pepe ${ }^{19}$, L. Perrone ${ }^{47}$, E. Petermann ${ }^{91}$, C. Peters ${ }^{39}$, S. Petrera ${ }^{48,49}$, Y. Petrov ${ }^{80}$, J. Phuntsok ${ }^{88}$, R. Piegaia ${ }^{3}$, T. Pierog ${ }^{36}$, P. Pieroni ${ }^{3}$, M. Pimenta ${ }^{67}$, V. Pirronello ${ }^{45}$, M. Platino ${ }^{8}$, M. Plum ${ }^{39}$, A. Porcelli ${ }^{36}$, C. Porowski ${ }^{65}$, R.R. Prado ${ }^{15}$, P. Privitera ${ }^{89}$, M. Prouza ${ }^{26}$, E.J. Quel ${ }^{2}$, S. Querchfeld ${ }^{34}$, S. Quinn ${ }^{78}$, J. Rautenberg ${ }^{34}$, O. Ravel $^{33}$, D. Ravignani ${ }^{8}$, D. Reinert ${ }^{39}$, B. Revenu ${ }^{33}$, J. Ridky ${ }^{26}$, M. Risse ${ }^{41}$, P. Ristori ${ }^{2}$, V. Rizi ${ }^{48}$, W. Rodrigues de Carvalho $^{77}$, J. Rodriguez Rojo ${ }^{9}$, M.D. Rodríguez-Frías ${ }^{75}$, D. Rogozin ${ }^{36}$, J. Rosado ${ }^{74}$, M. Roth $^{36}$, E. Roulet ${ }^{1}$, A.C. Rovero ${ }^{5}$, S.J. Saffi ${ }^{12}$, A. Saftoiu ${ }^{68}$, H. Salazar ${ }^{55}$, A. Saleh ${ }^{73}$, F. Salesa Greus ${ }^{88}$, G. Salina ${ }^{44}$, J.D. Sanabria Gomez ${ }^{24}$, F. Sánchez ${ }^{8}$, P. Sanchez-Lucas ${ }^{76}$, E.M. Santos ${ }^{16}$, E. Santos ${ }^{17}$, F. Sarazin ${ }^{79}$, B. Sarkar ${ }^{34}$, R. Sarmento ${ }^{67}$, C. Sarmiento-Cano ${ }^{24}$, R. Sato ${ }^{9}$, C. Scarso ${ }^{9}$, M. Schauer ${ }^{34}$, V. Scherini ${ }^{47}$, H. Schieler ${ }^{36}$, D. Schmidt ${ }^{36}$, O. Scholten ${ }^{62, b}$, H. Schoorlemmer ${ }^{90}$, P. Schovánek ${ }^{26}$, F.G. Schröder ${ }^{36}$, A. Schulz ${ }^{36}$, J. Schulz ${ }^{61}$, J. Schumacher ${ }^{39}$, S.J. Sciutto ${ }^{4}$, A. Segreto ${ }^{50}$, M. Settimo ${ }^{30}$, A. Shadkam ${ }^{83}$, R.C. Shellard ${ }^{13}$, G. Sigl ${ }^{40}$, O. Sima $^{70}$, A. Śmiałkowski ${ }^{66}$, R. Šmída ${ }^{36}$, G.R. Snow ${ }^{11}$, P. Sommers ${ }^{88}$, S. Sonntag ${ }^{41}$, J. Sorokin ${ }^{12}$, R. Squartini ${ }^{9}$, Y.N. Srivastava ${ }^{86}$, D. Stanca ${ }^{68}$, S. Stanič ${ }^{73}$, J. Stapleton ${ }^{87}$, J. Stasielak ${ }^{65}$, M. Stephan ${ }^{39}$, A. Stutz ${ }^{31}$, F. Suarez ${ }^{8,11}$, M. Suarez Durán ${ }^{24}$, T. Suomijärvi ${ }^{28}$, A.D. Supanitsky ${ }^{5}$, M.S. Sutherland ${ }^{87}$, J. Swain ${ }^{86}$, Z. Szadkowski ${ }^{66}$,

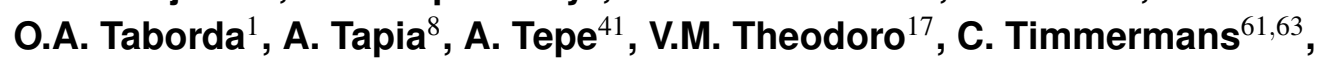
C.J. Todero Peixoto ${ }^{14}$, G. Toma ${ }^{68}$, L. Tomankova ${ }^{36}$, B. Tomé ${ }^{67}$, A. Tonachini ${ }^{46}$, G. Torralba Elipe ${ }^{77}$, D. Torres Machado ${ }^{22}$, P. Travnicek ${ }^{26}$, M. Trini ${ }^{73}$, R. Ulrich ${ }^{36}$, M. Unger ${ }^{85,36}$, M. Urban ${ }^{39}$, J.F. Valdés Galicia ${ }^{60}$, I. Valiño ${ }^{77}$, L. Valore ${ }^{43}$, 


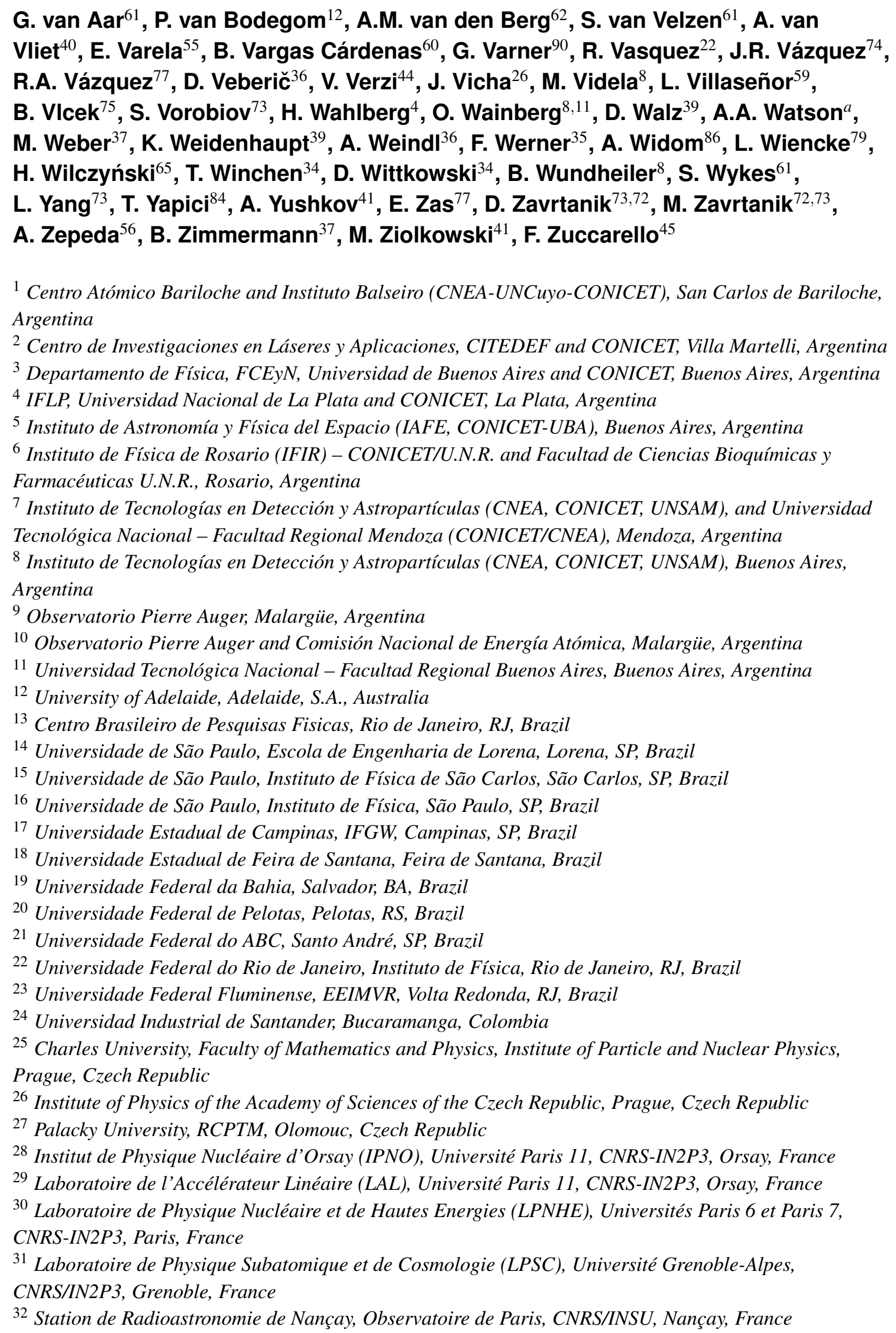

${ }^{2}$ Centro de Investigaciones en Láseres y Aplicaciones, CITEDEF and CONICET, Villa Martelli, Argentina

${ }^{3}$ Departamento de Física, FCEyN, Universidad de Buenos Aires and CONICET, Buenos Aires, Argentina

${ }^{4}$ IFLP, Universidad Nacional de La Plata and CONICET, La Plata, Argentina

${ }^{5}$ Instituto de Astronomía y Física del Espacio (IAFE, CONICET-UBA), Buenos Aires, Argentina

${ }^{6}$ Instituto de Física de Rosario (IFIR) - CONICET/U.N.R. and Facultad de Ciencias Bioquímicas y

Farmacéuticas U.N.R., Rosario, Argentina

${ }^{7}$ Instituto de Tecnologías en Detección y Astropartículas (CNEA, CONICET, UNSAM), and Universidad

Tecnológica Nacional - Facultad Regional Mendoza (CONICET/CNEA), Mendoza, Argentina

${ }^{8}$ Instituto de Tecnologías en Detección y Astropartículas (CNEA, CONICET, UNSAM), Buenos Aires,

Argentina

${ }^{9}$ Observatorio Pierre Auger, Malargüe, Argentina

${ }^{10}$ Observatorio Pierre Auger and Comisión Nacional de Energía Atómica, Malargüe, Argentina

${ }^{11}$ Universidad Tecnológica Nacional - Facultad Regional Buenos Aires, Buenos Aires, Argentina

${ }^{12}$ University of Adelaide, Adelaide, S.A., Australia

${ }^{13}$ Centro Brasileiro de Pesquisas Fisicas, Rio de Janeiro, RJ, Brazil

${ }^{14}$ Universidade de São Paulo, Escola de Engenharia de Lorena, Lorena, SP, Brazil

${ }^{15}$ Universidade de São Paulo, Instituto de Física de São Carlos, São Carlos, SP, Brazil

${ }^{16}$ Universidade de São Paulo, Instituto de Física, São Paulo, SP, Brazil

${ }^{17}$ Universidade Estadual de Campinas, IFGW, Campinas, SP, Brazil

${ }^{18}$ Universidade Estadual de Feira de Santana, Feira de Santana, Brazil

${ }^{19}$ Universidade Federal da Bahia, Salvador, BA, Brazil

${ }^{20}$ Universidade Federal de Pelotas, Pelotas, RS, Brazil

${ }^{21}$ Universidade Federal do ABC, Santo André, SP, Brazil

${ }^{22}$ Universidade Federal do Rio de Janeiro, Instituto de Física, Rio de Janeiro, RJ, Brazil

${ }^{23}$ Universidade Federal Fluminense, EEIMVR, Volta Redonda, RJ, Brazil

${ }^{24}$ Universidad Industrial de Santander, Bucaramanga, Colombia

${ }^{25}$ Charles University, Faculty of Mathematics and Physics, Institute of Particle and Nuclear Physics, Prague, Czech Republic

${ }^{26}$ Institute of Physics of the Academy of Sciences of the Czech Republic, Prague, Czech Republic

${ }^{27}$ Palacky University, RCPTM, Olomouc, Czech Republic

${ }^{28}$ Institut de Physique Nucléaire d'Orsay (IPNO), Université Paris 11, CNRS-IN2P3, Orsay, France

${ }^{29}$ Laboratoire de l'Accélérateur Linéaire (LAL), Université Paris 11, CNRS-IN2P3, Orsay, France

${ }^{30}$ Laboratoire de Physique Nucléaire et de Hautes Energies (LPNHE), Universités Paris 6 et Paris 7 , CNRS-IN2P3, Paris, France

${ }^{31}$ Laboratoire de Physique Subatomique et de Cosmologie (LPSC), Université Grenoble-Alpes, CNRS/IN2P3, Grenoble, France

32 Station de Radioastronomie de Nançay, Observatoire de Paris, CNRS/INSU, Nançay, France 
${ }^{33}$ SUBATECH, École des Mines de Nantes, CNRS-IN2P3, Université de Nantes, Nantes, France

${ }^{34}$ Bergische Universität Wuppertal, Fachbereich C - Physik, Wuppertal, Germany

${ }^{35}$ Karlsruhe Institute of Technology (KIT) - Campus South - Institut für Experimentelle Kernphysik (IEKP), Karlsruhe, Germany

${ }^{36}$ Karlsruhe Institute of Technology (KIT) - Campus North - Institut für Kernphysik (IKP), Karlsruhe, Germany

${ }^{37}$ Karlsruhe Institute of Technology (KIT) - Campus North - Institut für Prozessdatenverarbeitung und Elektronik (IEKP), Karlsruhe, Germany

${ }^{38}$ Max-Planck-Institut für Radioastronomie, Bonn, Germany

${ }^{39}$ RWTH Aachen University, III. Physikalisches Institut A, Aachen, Germany

${ }^{40}$ Universität Hamburg, II. Institut für Theoretische Physik, Hamburg, Germany

${ }^{41}$ Universität Siegen, Fachbereich 7 Physik - Experimentelle Teilchenphysik, Siegen, Germany

${ }^{42}$ Università di Milano and Sezione INFN, Milan, Italy

${ }^{43}$ Università di Napoli "Federico II" and Sezione INFN, Napoli, Italy

${ }^{44}$ Università di Roma II "Tor Vergata” and Sezione INFN, Roma, Italy

45 Università di Catania and Sezione INFN, Catania, Italy

${ }^{46}$ Università di Torino and Sezione INFN, Torino, Italy

${ }^{47}$ Dipartimento di Matematica e Fisica “E. De Giorgi” dell'Università del Salento and Sezione INFN, Lecce, Italy

${ }^{48}$ Dipartimento di Scienze Fisiche e Chimiche dell'Università dell'Aquila and Sezione INFN, L'Aquila, Italy

${ }^{49}$ Gran Sasso Science Institute (INFN), L'Aquila, Italy

${ }^{50}$ Istituto di Astrofisica Spaziale e Fisica Cosmica di Palermo (INAF), Palermo, Italy

51 INFN, Laboratori Nazionali del Gran Sasso, Assergi (L'Aquila), Italy

52 Osservatorio Astrofisico di Torino (INAF), Torino, Italy

53 INFN, Sezione di Torino, Italy

54 Università di Torino, Torino, Italy

${ }^{55}$ Benemérita Universidad Autónoma de Puebla, Puebla, México

56 Centro de Investigación y de Estudios Avanzados del IPN (CINVESTAV), México, D.F., México

${ }^{57}$ Unidad Profesional Interdisciplinaria en Ingeniería y Tecnologías Avanzadas del Instituto Politécnico Nacional (UPIITA-IPN), México, D.F., México

${ }^{58}$ Universidad Autónoma de Chiapas, Tuxtla Gutiérrez, Chiapas, México

${ }^{59}$ Universidad Michoacana de San Nicolás de Hidalgo, Morelia, Michoacán, México

${ }^{60}$ Universidad Nacional Autónoma de México, México, D.F., México

${ }^{61}$ IMAPP, Radboud University Nijmegen, Nijmegen, Netherlands

${ }^{62}$ KVI-Center for Advanced Radiation Technology, University of Groningen, Groningen, Netherlands

${ }^{63}$ Nikhef, Science Park, Amsterdam, Netherlands

${ }^{64}$ ASTRON, Dwingeloo, Netherlands

${ }^{65}$ Institute of Nuclear Physics PAN, Krakow, Poland

${ }^{66}$ University of Łódź, Łódź, Poland

${ }^{67}$ Laboratório de Instrumentação e Física Experimental de Partículas (LIP) and Instituto Superior Técnico, Universidade de Lisboa (UL), Portugal

68 “Horia Hulubei” National Institute for Physics and Nuclear Engineering, Bucharest-Magurele, Romania

${ }^{69}$ Institute of Space Science, Bucharest-Magurele, Romania

${ }^{70}$ University of Bucharest, Physics Department, Bucharest, Romania

${ }^{71}$ University Politehnica of Bucharest, Bucharest, Romania

72 Experimental Particle Physics Department, J. Stefan Institute, Ljubljana, Slovenia

${ }^{73}$ Laboratory for Astroparticle Physics, University of Nova Gorica, Nova Gorica, Slovenia

${ }^{74}$ Universidad Complutense de Madrid, Madrid, Spain 
${ }^{75}$ Universidad de Alcalá, Alcalá de Henares, Madrid, Spain

${ }^{76}$ Universidad de Granada and C.A.F.P.E., Granada, Spain

${ }^{77}$ Universidad de Santiago de Compostela, Santiago de Compostela, Spain

${ }^{78}$ Case Western Reserve University, Cleveland, OH, USA

${ }^{79}$ Colorado School of Mines, Golden, CO, USA

${ }^{80}$ Colorado State University, Fort Collins, CO, USA

${ }^{81}$ Department of Physics and Astronomy, Lehman College, City University of New York, Bronx, NY, USA

${ }^{82}$ Fermilab, Batavia, IL, USA

${ }^{83}$ Louisiana State University, Baton Rouge, LA, USA

${ }^{84}$ Michigan Technological University, Houghton, MI, USA

${ }^{85}$ New York University, New York, NY, USA

${ }^{86}$ Northeastern University, Boston, MA, USA

87 Ohio State University, Columbus, OH, USA

${ }^{88}$ Pennsylvania State University, University Park, PA, USA

${ }^{89}$ University of Chicago, Enrico Fermi Institute, Chicago, IL, USA

${ }^{90}$ University of Hawaii, Honolulu, HI, USA

${ }^{91}$ University of Nebraska, Lincoln, NE, USA

92 University of New Mexico, Albuquerque, NM, USA

${ }^{a}$ School of Physics and Astronomy, University of Leeds, Leeds, United Kingdom

${ }^{b}$ Also at Vrije Universiteit Brussels, Brussels, Belgium

E-mail: auger_spokespersons@fnal.gov

ABSTRACT: To exploit the full potential of radio measurements of cosmic-ray air showers at $\mathrm{MHz}$ frequencies, a detector timing synchronization within $1 \mathrm{~ns}$ is needed. Large distributed radio detector arrays such as the Auger Engineering Radio Array (AERA) rely on timing via the Global Positioning System (GPS) for the synchronization of individual detector station clocks. Unfortunately, GPS timing is expected to have an accuracy no better than about 5 ns. In practice, in particular in AERA, the GPS clocks exhibit drifts on the order of tens of ns. We developed a technique to correct for the GPS drifts, and an independent method is used to cross-check that indeed we reach a nanosecond-scale timing accuracy by this correction. First, we operate a "beacon transmitter" which emits defined sine waves detected by AERA antennas recorded within the physics data. The relative phasing of these sine waves can be used to correct for GPS clock drifts. In addition to this, we observe radio pulses emitted by commercial airplanes, the position of which we determine in real time from Automatic Dependent Surveillance Broadcasts intercepted with a software-defined radio. From the known source location and the measured arrival times of the pulses we determine relative timing offsets between radio detector stations. We demonstrate with a combined analysis that the two methods give a consistent timing calibration with an accuracy of $2 \mathrm{~ns}$ or better. Consequently, the beacon method alone can be used in the future to continuously determine and correct for GPS clock drifts in each individual event measured by AERA.

KEYWORDS: Pierre Auger Observatory; AERA; cosmic rays; extensive air showers; radio emission; ADS-B; time synchronization. 


\section{Contents}

1. Introduction 10

2. The AERA reference beacon 3

2.1. Setup description

2.2 Method and algorithm

2.2.1 Cross-correlation of the beacon signal 6

2.2.2 Practical implementation

2.3 Results of beacon analysis 8

3. Airplane calibration 9

3.1 Method description 9

3.2 The setup 11

3.3 Airplane calibration analysis 12

4. Combined timing analysis 16

4.1 One airplane 17

4.2 Multiple airplanes 18

4.3 Reconstruction improvement 19

5. Discussion 21

6. Conclusions 23

\section{Introduction}

In the last few years, radio detection of cosmic rays has matured from small prototype installations to full-fledged experiments contributing valuable information in the field of cosmic-ray physics [1]. In particular, radio detection has proven to be able to extract information on the energy $[2,3]$ and mass $[2,4-6]$ of the primary cosmic rays with a quality competitive with other detection techniques. To maximize the potential of radio detection arrays, a precise time synchronization of individual radio detector stations is needed. This is true in particular if interferometric analysis techniques are to be employed [7]. Another analysis strategy that requires very precise timing is the determination of the opening angle of the hyperbolic wavefront emitted by extensive air showers to deduce masssensitive parameters [8]. The consequence is that any radio detectors operating in the frequency band below $100 \mathrm{MHz}$ need a time synchronization on the order of $1 \mathrm{~ns}$ to exploit their full potential.

Nanosecond-scale timing synchronization has previously been achieved in cabled setups for radio detection of cosmic-ray air showers $[9,10]$. In fact, mature open-source solutions such as 


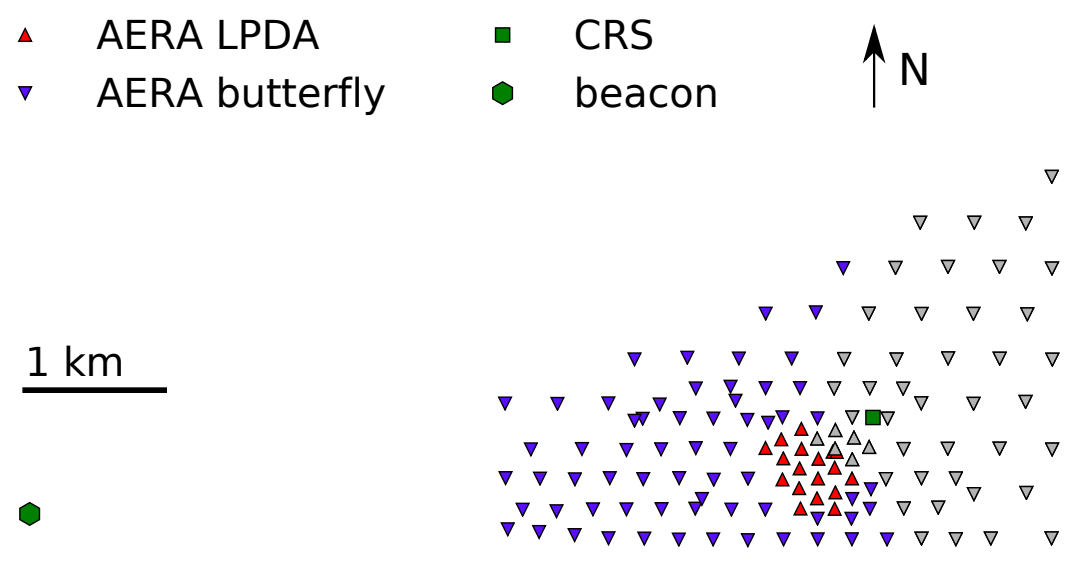

Figure 1. Map of the radio detector stations of the Auger Engineering Radio Array in the year 2014. Detector stations with either butterfly or logarithmic periodic dipole antennas (LPDAs) have been deployed [20]. The position of the beacon transmitter is west of the array, at the site of the 'Coihueco' fluorescence telescope building. Only the subset of radio detector stations capable of deep buffering for an external trigger, marked with red and blue triangles, was used for results presented in this paper (other stations are marked in gray). The ADS-B reception hardware is installed at the Central Radio Station (CRS) in the middle of AERA. Not shown here for simplicity: 25 extra antenna stations of butterfly type deployed in the south in 2015; Auger particle detectors at the same site (cf. Refs. [4,13]).

"White Rabbit" [11] have been developed in the past few years and are nowadays used by a broad community to reliably achieve sub-nanosecond timing synchronization in distributed detectors. However, these solutions rely on cabled links to distribute timing signals in a deterministic and stable way. The time synchronization of autonomous detector stations connected only via wireless communications such as those operated in AERA [12] (see Fig. 1) at the Pierre Auger Observatory [13] constitute a much more difficult challenge.

AERA consists of 153 autonomous stations featuring different spacings of $150 \mathrm{~m}$ to $750 \mathrm{~m}$ on an area of $17 \mathrm{~km}^{2}$. Each station features its individual solar power supply, and local data-acquisition with self-trigger on the radio signal. In addition, an external trigger by the Auger particle and fluorescence detectors is sent through the wireless communication to a part of the radio array. The same external trigger mechanism is also used to communicate a forced trigger at a rate of $0.01 \mathrm{~Hz}$ to each station, which provides a data set of coincident background measurements. For the proof-of-principle of the time synchronization methods presented here, only a part of the array is used, namely only those stations which feature an external trigger in addition to the self-trigger, and which have been commissioned before 2015. Later, the methods will be applied to the other stations. The used stations are marked with colored triangles in Fig. 11.

Time synchronization at AERA is performed via a GPS clock in each station, which is in principle capable of reaching an accuracy of $\sim 5 \mathrm{~ns}$ [14], in particular since equal cable lengths are used in all stations for all types of signal cables. In practice, however, it is often seen that this timing precision is only achieved over short time scales and that drifts of up to several tens of nanoseconds occur over the course of a day. This is true in particular for the individual radio detector stations of AERA. GPS-disciplined oscillators could possibly improve timing stability on scales of minutes to hours, and should be investigated as an option for future distributed radio 
detectors. For AERA, they were not considered for various reasons (focus on relative timing, cost issues, increased complexity and thus possibly higher rate of failure in the field).

To nevertheless reach the desired nanosecond-scale timing precision, alternative measures were employed within AERA. In particular, we operate a "beacon reference transmitter", originally pioneered in the context of the LOPES experiment [9] to achieve precise timing on an eventby-event basis. In this approach, sine waves at several frequencies are continuously emitted by a dedicated transmitter and recorded within the data stream of measured cosmic-ray events. Using the relative phases with which the different sine waves arrive at each individual AERA detector station, possible timing drifts of the station GPS clocks can be corrected for. However, one has to verify that systematic uncertainties, such as variable timing offsets introduced by changing ground and atmospheric conditions during the propagation of the beacon signal from the transmitter to the detector stations, are small compared to the desired accuracy of $1 \mathrm{~ns}$.

Therefore, an independent second method to perform a nanosecond-scale timing calibration of AERA detector stations was developed as a cross-check. It makes use of the fact that commercial airplanes emit radio pulses which can be seen in the physics data recorded by AERA. Such signals have previously been used in prototype setups of AERA to determine direction resolution and relative amplitude calibration [15]. In combination with real-time position information about the emitting airplane, which is available via digital ADS-B messages sent by the airplane, pulsed airplane signals can be used to independently check the time synchronization of the AERA detector stations and thus verify the validity of the drift correction performed with the beacon transmitter technique.

In this article, we first discuss the beacon transmitter technique in section 1 and the airplane timing analysis technique in section 3. Afterwards, we combine the two analyses in section 4 to cross-check them against each other and demonstrate that they give consistent results within $2 \mathrm{~ns}$, meaning in particular that the beacon technique is precise to at least $2 \mathrm{~ns}$. We finish with a discussion of remaining limitations of the techniques in section 5 and our conclusions in section 6 .

\section{The AERA reference beacon}

In this section we give technical details of the beacon transmitter, describe the algorithm used for monitoring and correction of the timing, and present example results for time drifts between AERA stations as observed with the beacon method.

\subsection{Setup description}

The AERA reference beacon is an emitter of continuous waves installed about $3 \mathrm{~km}$ west of the antenna array at the Coihueco telescope building of the Pierre Auger Observatory, which is located at a hill overlooking AERA (see Fig. I). The beacon system consists of a signal generator with amplifier connected via coaxial cable to a passive antenna emitting the signal. The signal generator mixes 4 sine wave signals generated by 4 Temperature Compensated Crystal Oscillators with a frequency stability of a few ppm. Several attenuators, filters and amplifiers make the overall amplitude as well as the strength of each individual signal adjustable, and suppress higher order artifacts due to non-linearities in the active electronic components. The resulting emitted signal contains the 4 desired sine waves transmitted by one single coaxial cable to the emitting antenna, and any 
other signals resulting from mixing or higher orders are suppressed by at least $40 \mathrm{~dB}$ and can be completely neglected.

The four frequencies have been chosen to lie inside the measurement band of $30-80 \mathrm{MHz}$ of the AERA detector stations: $58.887 \mathrm{MHz}, 61.523 \mathrm{MHz}, 68.555 \mathrm{MHz}$, and $71.191 \mathrm{MHz}$. The choice of the four frequencies has been done such that any three of them are sufficient to correct for timing drifts of at least $\pm 80 \mathrm{~ns}$, which had been observed in an earlier version of the AERA station electronics. In an ideal case without measurement uncertainties, two frequencies would suffice. Thus, the beacon system brings redundancy for signal disturbances by background.

The values of the frequencies are adapted to the sampling rates of the AERA stations (180 and $200 \mathrm{MHz}$ ) and the recorded trace length of at least 2048 samples such that they are almost an exact match to frequency bins of the Fourier transform of recorded AERA time traces. By this choice, artifacts such as aliasing are minimized. Moreover, the beacon frequencies can be filtered both in the local data acquisition of each antenna station as well as in offline data analysis. Hence, the beacon has minimal impact on self-triggering capabilities on air-shower pulses, and does not disturb the physics analyses.

The emitting dipole antenna is horizontally aligned, almost parallel to the north-south aligned antennas of the AERA stations. Thus, the beacon signal is mainly detected in the north-south channel of each AERA station, and only marginally visible in the east-west channel. Since there is only one GPS clock per station, performing the timing correction on only one channel is sufficient.

In addition to its capabilities for time calibration explained in the following, the beacon, as a known transmitter with well-defined properties, is a powerful tool for general monitoring of the detector performance. As side-remark we just report two cases in which the beacon was helpful to find and correct mistakes. During deployment of AERA we could quickly identify a few stations where accidentally the two channels had been switched, just by comparing the beacon amplitude in both channels. Also, a bug in the firmware of the stations caused a slightly worse timing precision for events triggered externally by the surface detector of the Pierre Auger Observatory compared to events self-triggered on radio pulses recorded by the antenna stations. Using the beacon analysis these problems were identified and could be solved.

\subsection{Method and algorithm}

The four sine waves emitted by the beacon superpose to a characteristic beat, which repeats approximately every $1.1 \mu$ s (see Fig. 2), i.e., a time series recorded by AERA of typical length $\geq 10 \mu \mathrm{s}$ contains several repetitions. During digital, offline analysis, the beacon beat can be isolated from other signals by applying digital filters to the beacon frequencies in the frequency spectrum, which is obtained by a Fourier transform of the time series recorded by the AERA antenna stations. The beacon transmitter thus does not degrade the quality of broad-band cosmic-ray radio pulses which AERA has been designed to measure.

Due to the different distances between the beacon and each of the antenna stations, the propagation time of the beacon signal $\tau_{\text {geo }}$ to each antenna is different. Since all positions have been measured with an accuracy of better than $10 \mathrm{~cm}$ (slightly worse for altitude) by differential GPS, the expected propagation times can be calculated with an accuracy of better than $1 \mathrm{~ns}$. Choosing one arbitrary station as a reference, it is easy to calculate the expected time difference $\Delta \tau_{\mathrm{geo}}$ of the 


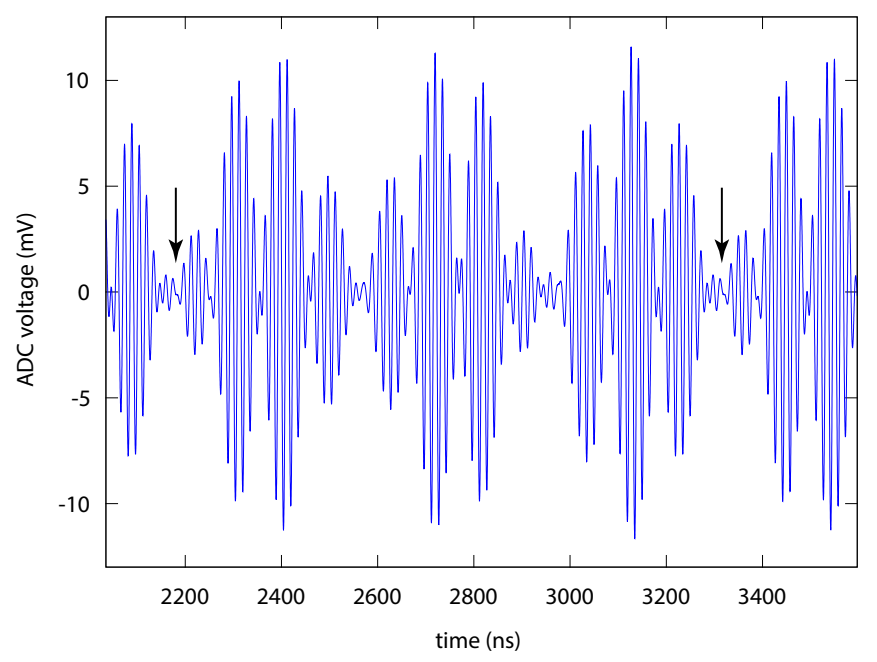

Figure 2. Beacon beat: a voltage-time trace recorded with the analog-to-digital converters (ADCs) of the north-south channel of an AERA station is digitally filtered such that it contains only the four beacon frequencies. The signal shape of the beacon beat has a periodicity of approximately $1100 \mathrm{~ns}$, as indicated by the two arrows.

beacon beat at each station with respect to this reference station (up to several $\mu$ s depending on the distance).

In reality, the measured time difference of the beacon beat deviates from the expected time difference by typically a few times $10 \mathrm{~ns}$ (cf. section 2.3). The reason for this is the drifting behavior of the GPS clocks. However, since the deviation is significantly smaller than the period of the beacon beat, it can be unambiguously determined and corrected for ${ }^{1}$. For each measured event a different, arbitrary station can be chosen as the reference, so that it is possible to determine the relative time offsets of the GPS clocks event-by-event and correct for them in offline analyses.

A question of practical relevance is the algorithm for the determination of these time differences between the beacon beat in different stations. A robust, but very time consuming way is provided by a cross-correlation analysis implemented in the offline analysis software. By an iterative search with sub-ns step size in a realistic range, e.g., $\pm 50 \mathrm{~ns}$ around the expectation, we determine which time shift between two stations maximizes the cross-correlation. This is the value by which the GPS time of a station has to be corrected to match that expected with the measured time of the beacon beat.

However, in practice, this procedure would dominate the total time needed for analyzing airshower events. Therefore, we use a slightly different method still relying on the same principle. The method used in our offline analysis exploits the phase differences of the beacon sine-wave signals. These phases are easy to measure, since the Fourier transform applied anyway during data analysis gives not only the amplitude, but also the phase at each frequency bin. Mathematically, the method relying on phase differences can be derived from the cross-correlation method in the following way.

\footnotetext{
${ }^{1}$ If fewer beacon frequencies were used, the beat period would be smaller, and thus the possible range for the correction of the GPS clock deviations would shrink.
} 


\subsubsection{Cross-correlation of the beacon signal}

Let $s_{1}(t)$ be the time series measured with antenna station 1 , and $s_{2}(t)$ the time series measured with antenna station 2 . The signal of station 2 is shifted by $\Delta \tau$ to compensate for the different arrival times of the beacon beat. Then, the cross-correlation $C C$ of both signals is ${ }^{2}$ :

$$
C C=\int s_{1}(t) s_{2}(t-\Delta \tau) \mathrm{d} t .
$$

We know that the beacon signal at the two stations is the sum of the four transmitted sine waves, which have different amplitudes $A_{i, j}$ and phases $\phi_{i, j}$ at each station, but the same frequencies $\omega_{j}(i=1,2$ is an index running over the stations; $j=1,2,3,4$ is an index running over the beacon frequencies):

$$
s_{i}(t)=\sum_{j} A_{i, j} \cdot \sin \left(\omega_{j} t+\phi_{i, j}\right) .
$$

Evaluating the cross-correlation integral, all products of sines with different frequencies are 0 , and only the products for sines with equal frequency remain:

$$
\begin{aligned}
C C=\sum_{j} \int A_{1, j} A_{2, j} \sin \left(\omega_{j} t+\phi_{1, j}\right) . \\
\cdot \sin \left(\omega_{j}(t-\Delta \tau)+\phi_{2, j}\right) \mathrm{d} t .
\end{aligned}
$$

As the absolute phase is not meaningful, only phase differences are determined experimentally and used in the calculation:

$$
\Delta \phi_{j}:=\phi_{2, j}-\phi_{1, j} .
$$

Finally, to find the values for $\Delta \phi_{j}$ and $\Delta \tau$ for which the cross-correlation is maximized, all constant factors can be neglected in the integral:

$$
\begin{aligned}
C C & =\sum_{j} \int A_{1, j} A_{2, j} \sin \left(\omega_{j} t\right) . \\
& \cdot \sin \left(\omega_{j}(t-\Delta \tau)+\Delta \phi_{j}\right) \mathrm{d} t \\
\propto & \sum_{j} A_{1, j} A_{2, j} \cos \left(\Delta \phi_{j}-\omega_{j} \Delta \tau\right),
\end{aligned}
$$

which holds exactly when the integration time is an integer multiple of the periods of all beacon frequencies, or approximately when the integration time is large compared to the period of the smallest frequency.

This equation can be interpreted in physical terms. The cross-correlation is maximum when the measured phase differences between the two stations at all beacon frequencies correspond exactly to what is expected from the time difference of the beacon beat at the two stations. Moreover, the different beacon frequencies receive a weight according to the amplitudes measured by the stations.

Since the amplitudes $A_{i, j}$ and the phase differences $\Delta \phi_{j}$ are measured quantities, the only variable in equation 2.5 is the time shift $\Delta \tau$. A starting guess for $\Delta \tau$ is $\Delta \tau_{\text {geo }}$, which is the geometrically expected difference in the propagation time of the beacon signal. Afterwards, we search for the $\Delta \tau_{\max }$ providing the global minimum of equation 2.5 in a range of $\pm 100 \mathrm{~ns}$ around the expectation $\Delta \tau_{\text {geo }}$. The difference between $\Delta \tau_{\max }$ and $\Delta \tau_{\text {geo }}$ is interpreted as current offset of the GPS clocks relative to each other, and used as correction value.

\footnotetext{
${ }^{2}$ In practice, the integral is calculated as a discrete sum with boundaries, which is unimportant for the theoretical treatment here.
} 


\subsubsection{Practical implementation}

In practice two major problems occur, which are not reflected by the pure mathematical derivation, but are taken into account for the implementation of the beacon method.

First, the phase measurements are affected by noise. Since the orientation of the electric field vector is random for noise, thus, it generally points in a direction different from that of the electric field vector of the signal. Consequently, the random phase of noise changes the measured phase of the signal. For ns-timing precision, a maximum phase error of $\lesssim 20^{\circ}$ can be tolerated. The $20^{\circ}$ correspond to a shift of slightly less than $1 \mathrm{~ns}$, since a full period $\left(\widehat{=} 360^{\circ}\right)$ is about $17 \mathrm{~ns}$ for the lowest beacon frequency. On the basis of simple trigonometry it is possible to estimate the minimum signal-to-noise ratio, which is tolerable in order to avoid phase errors of more than $20^{\circ}$ : the phase error is maximum, when the electric field vector of the noise is perpendicular to the electric field vector of the beacon signal. Then the phase error is $\arctan (N / S)$ with $S$ and $N$ the lengths of the signal and noise vectors, respectively, which requires the signal amplitude to be $\tan 20^{\circ}=2.75$ times larger than the noise amplitude. As usual in radio engineering, the signal-to-noise ratio is defined in the power domain (amplitudes squared), such that the threshold for the signal-to-noise ratio is $2.75^{2} \approx 8$. In the case of the AERA beacon, the emission power has been set to exceed this threshold. In fact, with the exception of a few measurements with exceptionally strong background fluctuations, the signal-to-noise ratio is much larger ( $>100$ for stations close to the beacon). Then, systematic uncertainties dominate, which have not been investigated in detail, since they appear to be unimportant for the desired ns-timing precision. Thus, weighting the different beacon frequencies by their amplitude as in equation 2.5 is unreasonable. Consequently, the weighting by the amplitudes is replaced by a weighting with the signal-to-noise ratio, capping the weight to a maximum value for any signal-to-noise ratio $\geq 10$.

Second, even for very large signal-to-noise ratios the phase is not completely determined by the geometrical propagation of the beacon signal. Ground properties as well as the antenna characteristics influence the measured phases. These effects are very hard to calculate or simulate, since the beacon signal propagates nearly horizontally, parallel to the ground. Thus, the ground is within the first Fresnel zone of the signal and cannot be neglected. Therefore, usual free-space approximations in the antenna simulations do not hold. Moreover, measurements of the group delay are cumbersome, and have been done only for selected arrival directions to cross-check the antenna simulations [20]. Generally, the used antenna model compares well to the measurement, but with larger deviations for near-horizontal signals. In particular, the simulated group delays of the antennas deviate significantly from measurements for the zenith angle of the beacon signals, which is $\theta \approx 89^{\circ}$ decreasing slightly with increasing distance of the antennas to the beacon.

Hence, in the actual timing correction performed event-by-event during analysis, the measured phases are not compared to the calculated expectation $\omega_{j} \Delta \tau_{\mathrm{geo}}$, but instead to reference values derived from phases measured by the real antenna stations. These reference values are obtained by averaging over approximately 100 triggered AERA events corresponding to the statistics of several hours. The measured reference values are in agreement with the geometrically calculated values. However, for some stations and frequencies the deviation is slightly larger than the desired accuracy of $1 \mathrm{~ns}$. Consequently, it is indeed necessary to use the measured reference values instead of the calculated ones. 


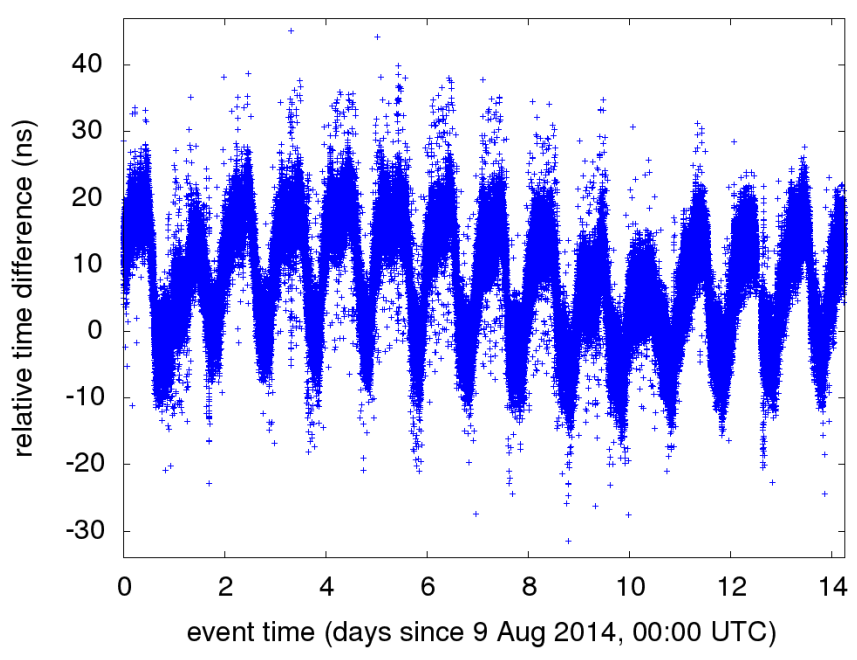

Figure 3. Example of the relative timing between two AERA stations determined with the beacon method. Each entry denotes the size of the timing correction between two typical AERA stations in one measured event, interpreted as a time offset between the GPS clocks of the two detector stations. The few outliers have not been investigated in detail, since the focus of the present analysis is on the proof-of-principle and the general behavior of the relative timing. A possible explanation for the outliers might be mis-measurements, e.g., because of occasional high background disturbances.

\subsection{Results of beacon analysis}

In the standard analysis pipeline of AERA, the beacon correction is automatically applied for all recorded events. This means that the timing of AERA stations participating in the event is shifted by the correction value obtained from the beacon analysis. A statistical study for many events of the amount by which one station has to be shifted against a specific other station reveals the relative timing accuracy and stability. To enhance the statistics, the beacon timing correction is not only applied to cosmic-ray candidate events, but also on background data triggered periodically at least every $100 \mathrm{~s}$, and we checked that the timing of cosmic-ray events is not systematically different from that of background measurements.

Fig. 3 shows a graphical representation of such a statistical study of the relative timing between two stations over several days. At a random point in time, the time offset between two AERA stations can be as large as several times $10 \mathrm{~ns}$. This would dominate the total timing accuracy and spoil any interferometry or wavefront analysis, if not corrected for. Moreover, the time offset between two stations experiences systematic drifts on time scales of several hours. The relative timing is stable only over short periods of a few minutes at a level of a few ns, corresponding to the nominal precision of the GPS clocks. However, even over these short time periods two GPS clocks of two different detector stations can still have an arbitrary offset of a few times $10 \mathrm{~ns}$.

The reason for these drifts and offsets is not known. The day-night structure in Fig. 3 indicates that environmental effects, like temperature, play a role, but they cannot fully explain the drifts. We performed several cross-checks to exclude possible explanations. In particular, the effect cannot be explained by the accuracy of the location stored in the GPS clocks. In laboratory measurements we connected two equal clocks to the very same antenna, and still observed qualitatively the same 
drifts, though on a slightly smaller scale, but still exceeding the $5 \mathrm{~ns}$ accuracy claimed by the manufacturer. This also excludes any changes of environmental conditions as the sole reason for the drifts, since both clocks have been exposed to exactly the same conditions in the laboratory. Thus, environmental effects in the field apparently do not cause the drifts, but enhance their size, which would explain the rough day-night structure in the offsets. The size of the drifts in the AERA stations was successfully reduced, by the installation of GPS antennas with higher gain, indicating that the signal-to-noise ratio of the received GPS signals plays a role (the results in Fig. 3 are with the new GPS antennas). Also the electronics of the local data acquisition seem to influence the size of the drifts, possibly by introducing some local interference. It seems that a complex combination of effects causes the observed drifts.

Fortunately, the GPS clock drifts can be corrected for in the offline analysis of measured events, regardless of their physical origin. However, we have to independently verify whether the drifts determined and corrected for by the beacon are indeed intrinsic to the GPS clocks and not caused by some external effect such as environmental influence on the propagation delay of the beacon signals. To verify the beacon correction, and to cross-check its finally achieved accuracy, we thus developed an independent time calibration using airplane signals described in the next section.

\section{Airplane calibration}

In this section, we describe the general concept, the hardware and software setup, and the analysis strategy for the timing calibration using commercial airplane signals.

\subsection{Method description}

To use a source for timing calibration, two requirements need to be fulfilled. First, the source must emit a signal that can be identified in the data taken by the radio detector array, simultaneously in as many detector stations as possible. A short, bandwidth-limited pulse is ideal as it can be timetagged precisely. Moreover, the position of the source needs to be known at the time of emission. From the known position and the relative arrival time of the signals in different detector stations, the time offsets between different detector stations can then be determined provided that the model for the wavefront used for the reconstruction is well adapted to the actual wavefront.

These requirements are fulfilled for commercial airplanes. Surprisingly, (some) commercial airplanes emit radio pulses in the $30-80 \mathrm{MHz}$ frequency range covered by AERA. The origin of these radio pulses is unclear. In 2011, using an upgraded version of a small prototype radio array at the Pierre Auger Observatory [16], we regularly observed airplane signals in the frequency band 30-260 MHz. However, the characteristics of these signals varied from one airplane to another. In some cases, the signals were periodic with a very high level of accuracy (repetitive bunches of 4 transients separated by $132870 \pm 90 \mu$ s followed by a pause of $861120 \pm 280 \mu \mathrm{s}$ ). In other cases, the arrival times of the signals did not exhibit any clear structure. Similar observations were made in CODALEMA. Possible sources of emission could be the TCAS (Traffic Collision Avoidance System, periodic emission), the ADS-B [17] transponder (periodic emission), the airplane navigation lights (periodic) or the DME (Distance Measuring Equipment, aperiodic) [18]. No definitive 


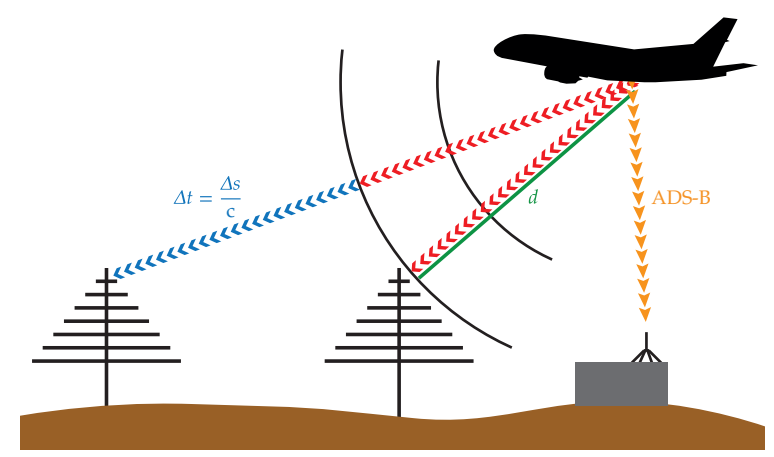

Figure 4. Concept of the time calibration using commercial airplanes. The airplane broadcasts its position via digital ADS-B packets at $1090 \mathrm{MHz}$. These signals are received and interpreted by a dedicated setup in the AERA field in real time. In addition, (some) commercial airplanes emit pulsed signals in the frequency range of 30-80 $\mathrm{MHz}$ recorded by the AERA detector stations.

answer could be found as the next step for determining the source would require classified information that we could not obtain. It is, however, not necessary to know the mechanism for the emission or the exact characteristics of the radio pulses to use them for timing calibration.

The position of an airplane can be readily determined in real time from digital broadcasts that modern airplanes emit at rates of $0.5-1 \mathrm{~Hz}$, via the ADS-B service. These digital broadcasts at a frequency of $1090 \mathrm{MHz}$ contain information such as an airplane-specific call-sign, longitude and latitude, altitude, velocity and heading of the airplane. They are intended to inform other aircraft and air traffic control in real time of airplane positions, but can indeed be received easily with equipment costing less than 20 USD by any interested person. All that is needed is reception hardware for the frequency of $1090 \mathrm{MHz}$ which can be used as "software-defined radio", meaning that demodulation, decoding and interpretation of the received signals are performed in software on a computer. Inexpensive receivers with a USB interface originally intended for the reception of digital TV signals (DVB-T) suit this purpose. There is a broad community employing such USB receivers with RealTek RTL2832U chipsets for the reception of various analog and digital signals under the term "RTL-SDR".

By combining the real-time position information from ADS-B data and the radio pulses emitted by the airplane as recorded in the AERA data stream, time offsets between GPS clocks of individual AERA stations can be calculated. The overall concept is visualized in Fig. 7 . Using the known position of both the airplane (interpolated from the ADS-B messages to the exact time of the event) and the AERA detector stations, the expected arrival times of the radio pulses can be calculated. The typical values are on the order of several $10 \mu \mathrm{s}$, since the typical distances are on the order $10 \mathrm{~km}$ (explanation in more detail follows later). Deviations from these expected arrival times can be interpreted as timing drifts between pairs of detector stations.

An additional complication that needs to be addressed is that the AERA central data acquisition system, which triggers on radio pulses detected by AERA stations as well as information from external detectors, has been optimized to suppress anthropic radio sources. In particular, it blinds regions of the sky from which radio pulses have been received repeatedly within a few minutes. Airplanes crossing the sky over the course of a few minutes constitute such sources and would thus 


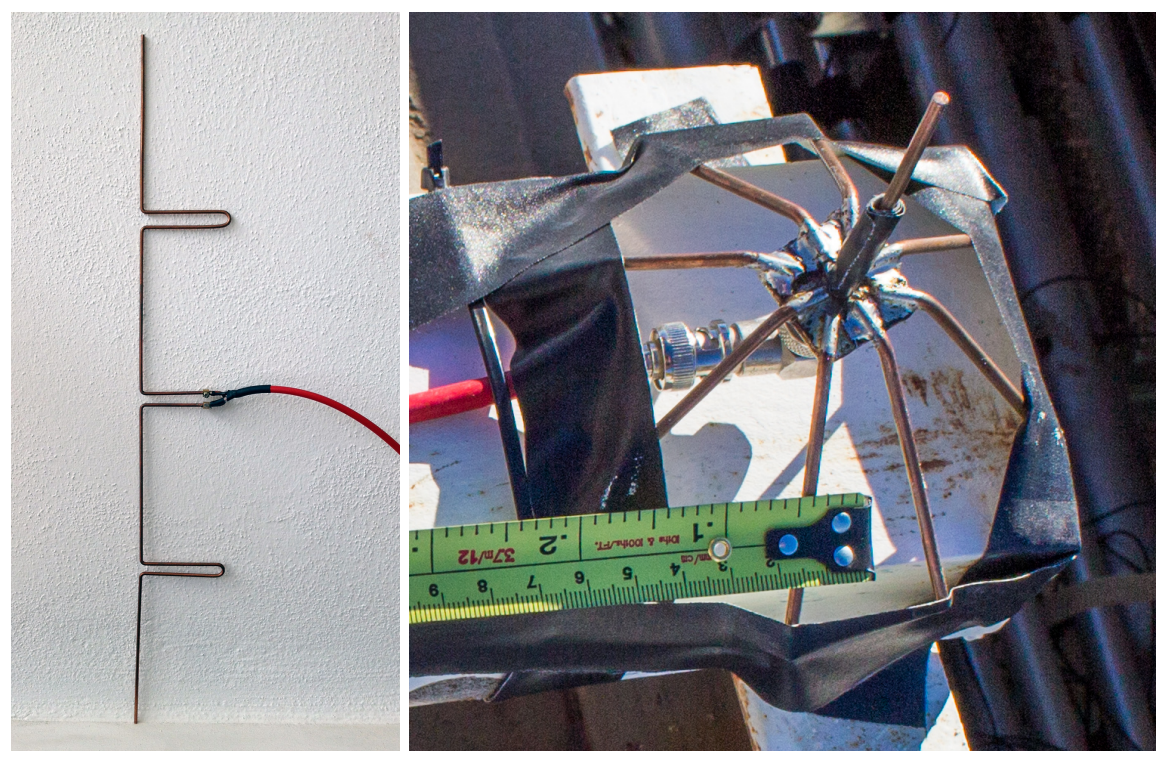

Figure 5. ADS-B antennas of two different types: four-segment collinear antenna (left) and ground-plane antenna (right). The ground-plane antenna was deployed in the AERA field.

be filtered out by our data acquisition. Therefore, the data acquisition software had to be modified, as will be described below.

\subsection{The setup}

A simple hardware setup was deployed at the Central Radio Station of AERA (cf. Fig. (1) to record the ADS-B information transmitted by airplanes in real time. It consists of a tailor-made antenna for the $1090 \mathrm{MHz}$ ADS-B signals, a USB receiver connected to a laptop and dedicated software to use the receiver as a software-defined radio decoding and processing the ADS-B data packets. We will shortly describe the individual items in the following.

After initial tests with a simple monopole antenna, two antennas optimized for $1090 \mathrm{MHz}$ signals were built and tested. The two types, a four-segment collinear antenna and a ground-plane antenna are depicted in Fig. 5. The latter antenna was built according to a design published by hobby enthusiasts ${ }^{3}$. Tests conducted at the Karlsruhe Institute of Technology (Fig. 6) showed that the ground-plane antenna reproducibly sees airplanes to further distances than the collinear antenna (or a simple monopole, not shown here). It was thus chosen as the antenna that was deployed on the roof of the Central Radio Station within the AERA field. In spite of its improvised design, the reception quality of the ground-plane antenna fully satisfied our needs; we only need to reliably detect airplanes in close vicinity to AERA. Further optimizations were thus not carried out.

The ground-plane antenna installed in the AERA field was connected to a USB receiver with a RealTek RTL2832U chipset and a Rafael Micro R820T tuner chip which can be tuned to $1090 \mathrm{MHz}$. This USB receiver, connected to a commercial laptop, has been working reliably over months of continuous operation.

\footnotetext{
${ }^{3}$ http://www.diylightanimation.com/wiki/images/0/01/ DIY_Quarter_Wave_Antenna.pdf
} 

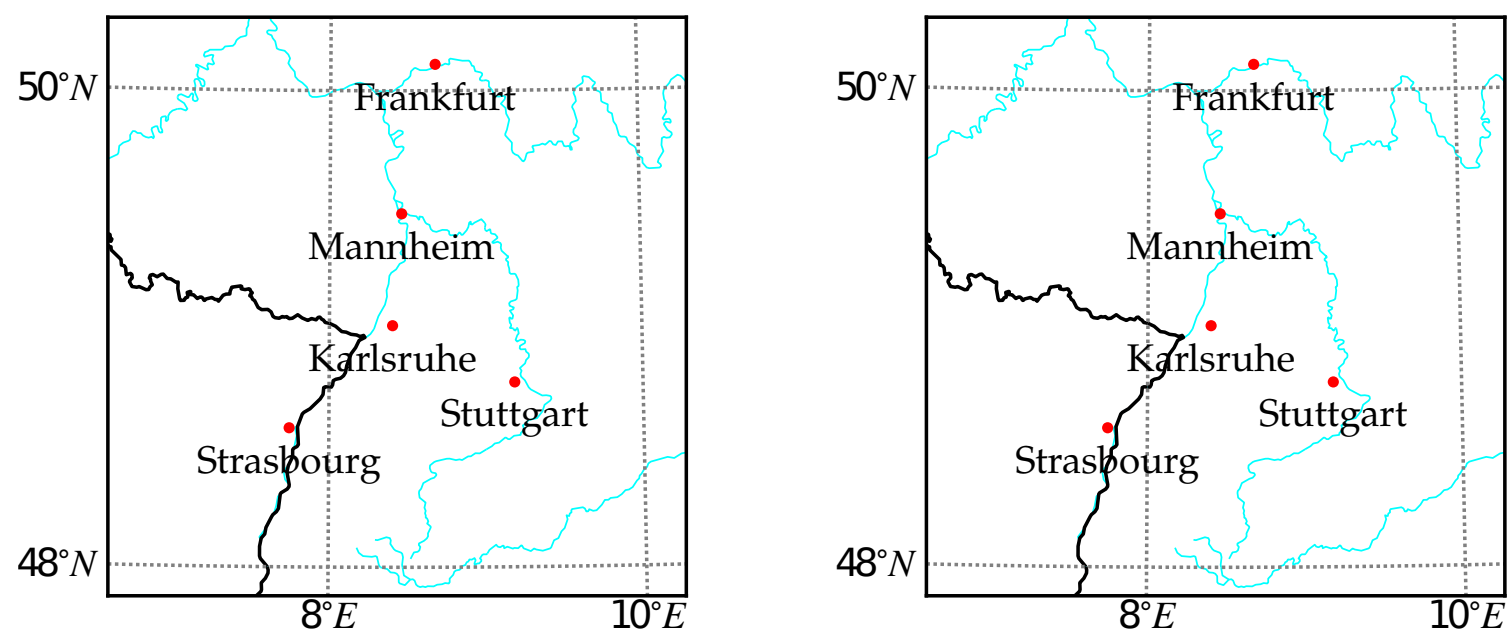

Figure 6. Reception area (in green) of the two ADS-B antenna tested at Karlsruhe Institute of Technology: Four-segment collinear antenna (left) and ground-plane antenna (right). Each single green point denotes a received airplane position. The western hemisphere was shielded by a concrete building.

For the decoding and interpretation of the ADS-B data packets, the open-source software package "dump1090" was extended for our needs. In addition to decoding the position and velocity information of airplanes for which ADS-B packets have been received, the software identifies airplanes that come close enough to AERA to be visible at a zenith angle up to $80^{\circ}$ from the vertical. While an airplane fulfills this condition, notification packets detailing the relevant information are sent in real time to our data acquisition software which then accepts radio triggers from a window of $5^{\circ}$ around the known airplane position instead of suppressing these radio pulses of clearly anthropic origin. In parallel, a log file with decoded ADS-B information such as latitude, longitude and altitude is stored on disk for easy follow-up analyses. The ADS-B messages do not contain reliable information on the position uncertainty, but our analysis itself confirms that the information is accurate enough to apply the presented method (cf. the cross-check of the beacon and airplane methods in section (1).

\subsection{Airplane calibration analysis}

Here, we explain how the analysis of airplane signals is carried out to deduce the time offsets of GPS clocks in individual radio detector stations with respect to one reference station.

When the central data acquisition is notified of an approaching airplane, it will let radio pulses from corresponding arrival directions trigger the readout of all detector stations, marking the corresponding events with a special "airplane trigger" flag. This means that airplane signals are detected by the same self-trigger algorithm also used for air showers, and can be separated from the normal AERA data stream using this flag .

We apply an iterative direction reconstruction [19] to these AERA events. We require simultaneous detection in at least 10 radio detector stations with a signal-to-noise ratio (defined as peak power divided by RMS noise power) of at least 15 . In addition to the arrival direction, we reconstruct a source distance using a spherical wavefront as adequate for a point-like source and exclude

\footnotetext{
${ }^{4}$ https://github.com/MalcolmRobb/dump1090
} 
events $^{5}$ with a wavefront radius of curvature of less than $7.5 \mathrm{~km}$ (commercial airplanes travel at altitudes of $10 \mathrm{~km}$ or more) or more than $99 \mathrm{~km}$. A typical airplane pulse as seen in one radio detector station is shown in Fig. 7 . We apply an upsampling procedure and a Hilbert envelope to the electric field trace reconstructed from the voltage traces of the east-west and north-south detection channels, respectively [19], and use the time of the maximum of the enveloped signal as the pulse arrival time in the reconstruction.

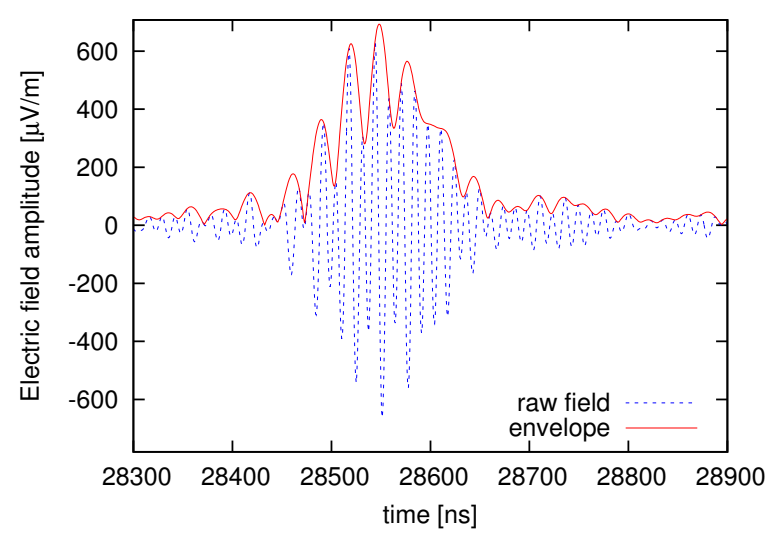

Figure 7. Typical radio pulse received from an airplane in the north-south component of the reconstructed electric field. A Hilbert envelope is calculated to read off the time of the pulse maximum. Time zero corresponds to the start of the recorded time trace, which has a total length of 50 microseconds.

An overview of a typical airplane event is shown in Fig. 8, illustrating that the radio pulses from airplanes trigger almost all radio detector stations simultaneously. (The radio stations in the eastern part were being read out with a separate data acquisition system not supporting the airplane trigger.) The color code denotes the relative arrival time of the radio pulses at the individual detector stations; in this case it is obvious that the source must be south-east of the array. In Fig. Q we illustrate the reconstructed arrival directions of all airplanes which have been seen in at least 10 AERA events within our data set from June to October 2014.

For airplane events reconstructible using AERA data, the corresponding recorded ADS-B data are analyzed. A unique airplane can be identified as the source of each detected AERA event. A total of 21 individual flights passing over AERA have been identified. Details are listed in table 1 in the appendix. Some airplanes, uniquely defined by their identification code, the so-called Mode-S call-sign, have been spotted several times in different fly-overs.

The ADS-B data for each airplane provide position information also for those parts of the airplane trajectory for which no AERA triggers were generated (be it because there were no radio pulses or because they came from directions with zenith angles above $80^{\circ}$, for which the data acquisition was not accepting airplane triggers). An example airplane trajectory deduced from ADS-B data is shown in Fig. 10. There is good agreement between the arrival direction recon-

\footnotetext{
${ }^{5}$ The wavefront reconstruction is applied to AERA raw data without any prior correction of detector station time offsets and is thus affected by GPS clock drifts. However, we only use the reconstructed distance to cut away rare radio-frequency interference pulses clearly not originating from airplanes, not for the determination of time offsets. The good agreement of the direction reconstructed from AERA events and ADS-B data illustrates that the resulting sample of "airplane events" is very clean.
} 


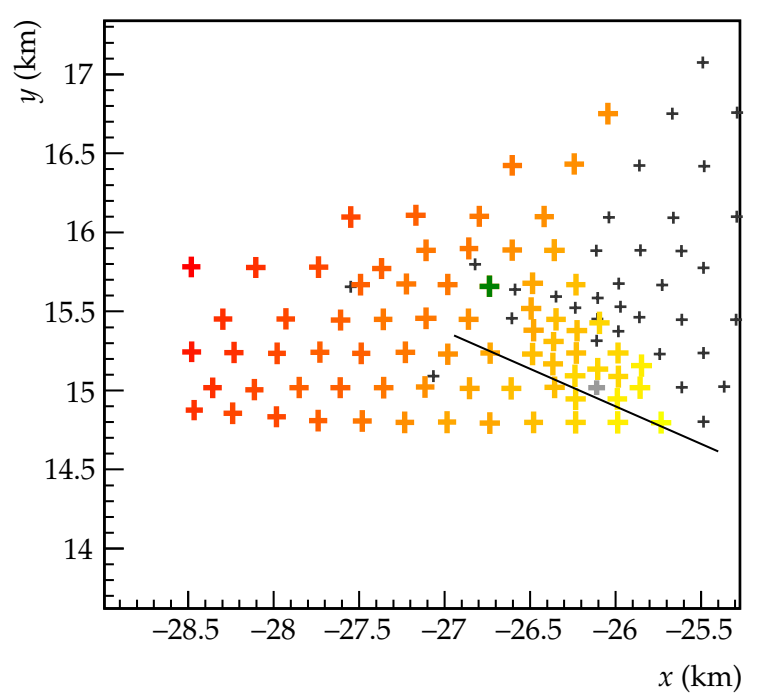

Figure 8. Typical airplane event as shown in the Offline EventBrowser. Every station is indicated by a cross. One station without detectable signal is marked with a gray cross, stations that are not in data acquisition are shown in black. Stations with a signal have a color indicating the arrival time from early (yellow) to late (red). The reconstructed arrival direction is marked with a black line. The station used as reference station in the analysis (station 40) is marked in green.

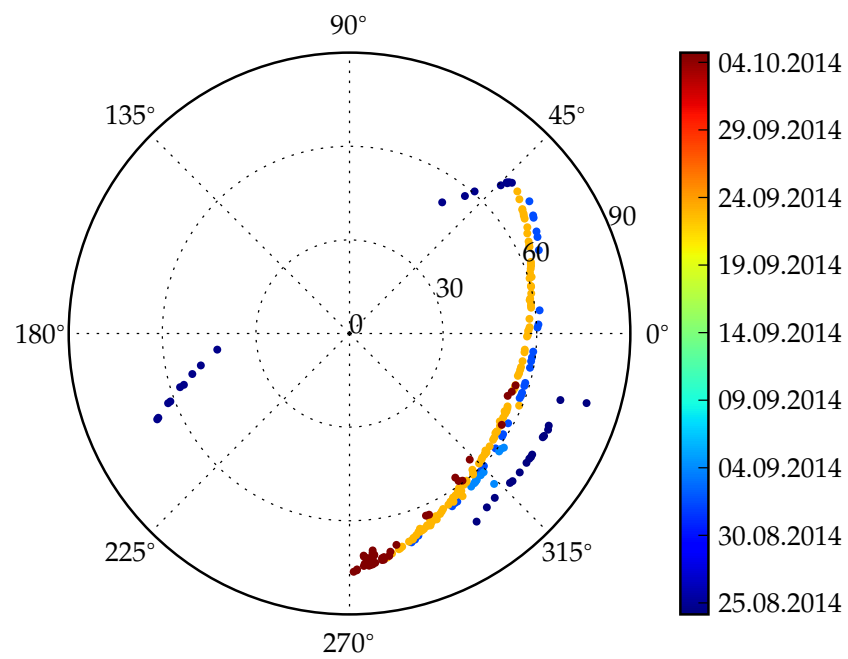

Figure 9. Reconstructed arrival directions of AERA events for airplane fly-overs with at least 10 successful detections. The sky map has the zenith at the center (cf. table 1 in the appendix) and East at $0^{\circ}$. The color of the dots represents the date of detection. For some airplanes a clear trajectory is visible.

structed from the AERA data (30-80 MHz) and the position information digitally broadcast by the airplane via ADS-B packets (1090 MHz), as shown for the same airplane in Fig. 11. The directions reconstructed from AERA data and calculated from ADS-B position data match generally within $0.3^{\circ}$. Also, the source distance derived from the spherical wavefront fit (not shown here) is in good agreement with the distance calculated from the ADS-B position data. (The source distance reconstructed from AERA data is only used as a cross-check here. For further analysis, only the ADS-B position is used.) 


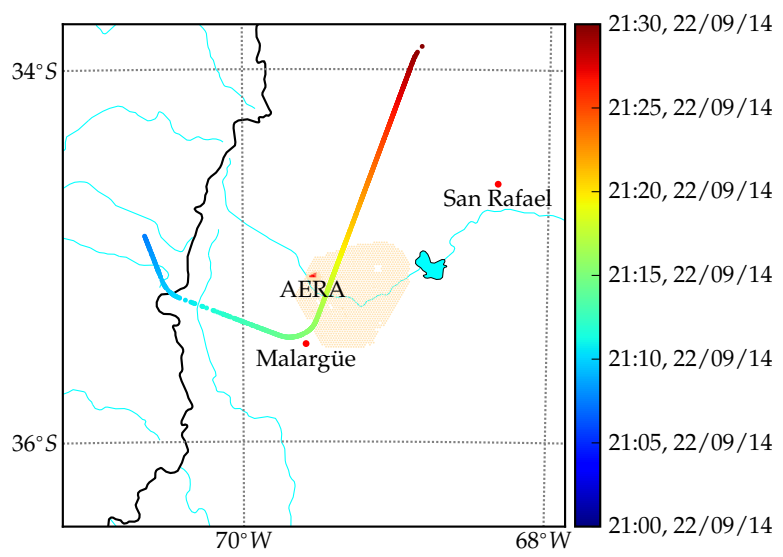

Figure 10. Trajectory of the airplane passing the AERA field (marked in red) and the main surface detector array of the Pierre Auger Observatory (orange shaded area) on September 22, 2014 at around 21:15 UTC based on ADS-B data.
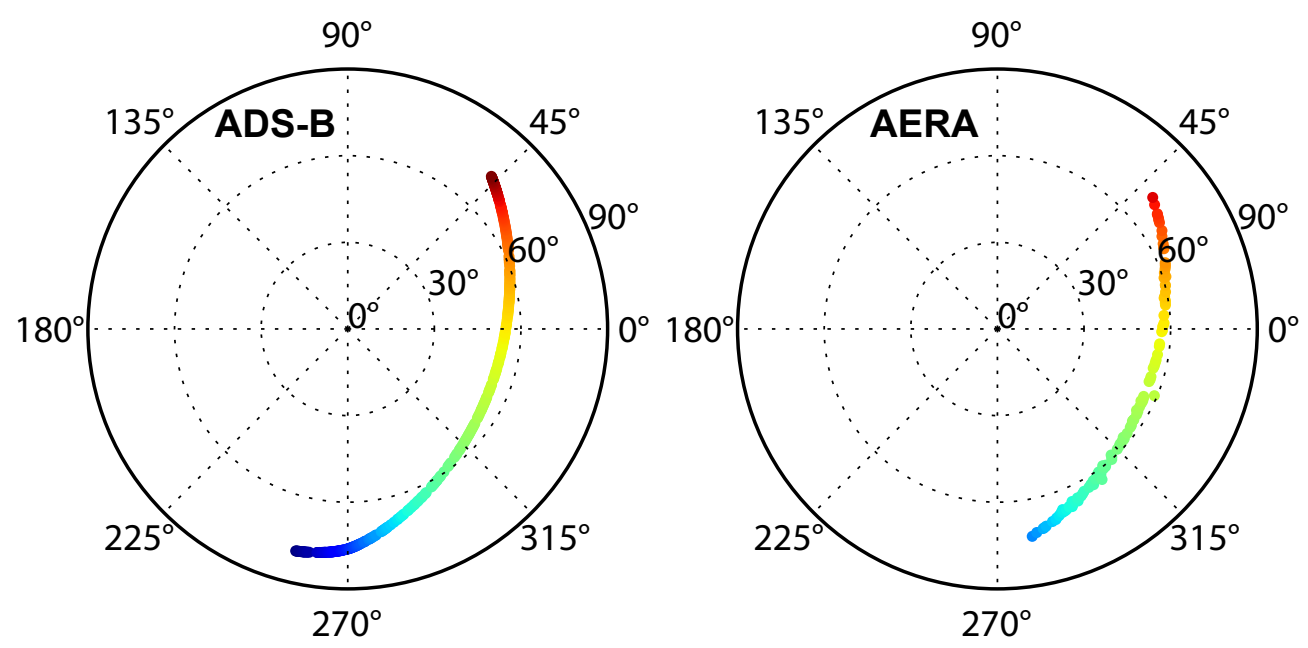

$21: 20$

21:19

Figure 11. Position of the airplane passing nearby AERA on September 22 based on ADS-B data (left) and reconstructed arrival directions from 115 AERA events of the same airplane (right). The mean deviation between the direction reconstructed with AERA and taken from the ADS-B data is $0.3^{\circ}$. The color code denotes the UTC time at which the airplane event was detected.

The ADS-B position data is only available at discrete times, typically with a rate of $0.5-1 \mathrm{~Hz}$. Therefore, the ADS-B position data are linearly interpolated in latitude and longitude. Surprisingly, this yields better consistency with the positions reconstructed from AERA data than an interpolation also taking into account the velocity and heading information available from the ADS-B data. The ADS-B position data is not only used for the calculation of the expected pulse arrival times, but is also used to determine an individual direction per AERA detector station for the application of the antenna pattern. (The airplane is at a typical distance of 10 to $30 \mathrm{~km}$, i.e., it is seen at angles differing on the order of $5-10^{\circ}$ for the different station of the array.)

As only relative time differences between AERA detector stations can be determined, one detector station is chosen as the reference (which by definition has a time offset of zero). The 


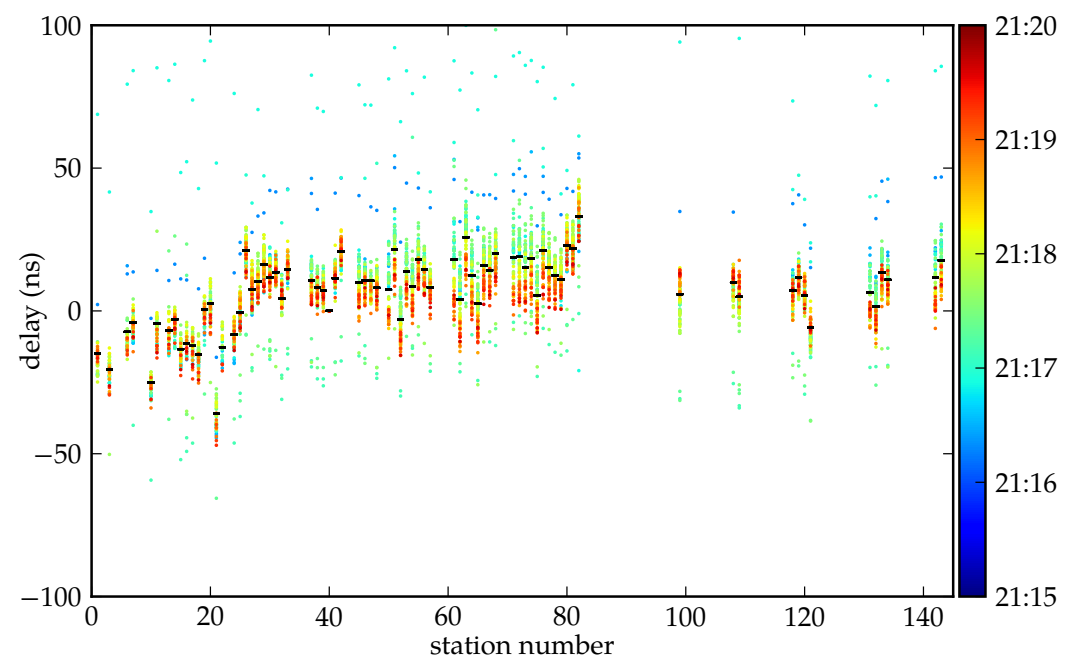

Figure 12. Per-event time offsets for all AERA detector stations determined from AERA and ADS-B data for an airplane detected on September 22. Time offsets are relative to reference station 40. Stations 1-24 are equipped with logarithmic-periodic dipole antennas, stations with numbers 25 and higher are equipped with butterfly antennas. Gaps in the station numbering are related to stations not participating in the airplane trigger (marked gray in Fig. (1). The color code denotes the local time at which the airplane event was detected.

reference station is chosen such that it is near the geometric center of the radio detector array and that it has a positive pulse detection in a large number of the AERA events for a given airplane.

The light travel time from the airplane to each antenna is calculated assuming that the refractive index of the atmosphere has a constant value of $n \approx 1.00024$ as that corresponding to the altitude of AERA (1,560 m above sea level). The calculated arrival times are then compared with the actual measured pulse arrival times with respect to the chosen reference station. The difference between the measured and predicted arrival times is the time offset of the individual detector station with respect to the reference station.

The result of this analysis for the example airplane already presented in Figs. 10 and 11 is depicted in Fig. 12. A number of features can be identified in the resulting time offsets. The individual offset values determined from the different AERA events in which the airplane was detected have a scatter of typically less than $10 \mathrm{~ns}$, but there are significant outliers. Within the scatter of $\approx 10 \mathrm{~ns}$, there is a time-ordering, i.e., there must be some systematic effect. We will discuss both aspects at a later point. The mean values calculated from the distributions are typically within $20 \mathrm{~ns}$ of the zero-line (which would correspond to perfect time synchronization of the array). This is approximately the scale of the drifts previously observed by the beacon analysis.

\section{Combined timing analysis}

With both the beacon timing calibration and airplane timing analysis in place, the two methods can now be combined to check them for consistency. If the beacon correction can indeed detect and mitigate timing drifts in the GPS clocks of the different AERA stations, then a follow-up airplane analysis should yield vanishing time offsets for all stations to the reference station. 


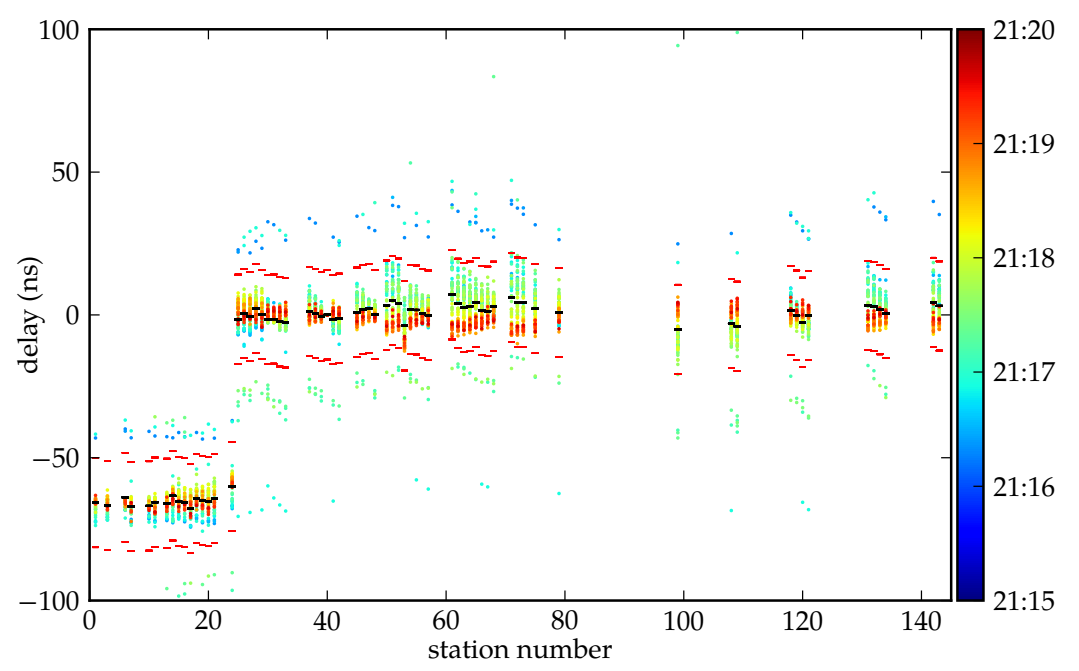

Figure 13. Same as Fig. 12 when applying the airplane timing calibration after per-event clock-drift correction using the beacon method. The red lines show the range of mean $\pm 4 \cdot \mathrm{MAD}$ (median absolute deviation).

\subsection{One airplane}

We first present the results of the combined beacon and airplane analysis inferred for the particular airplane discussed in the previous section. For the AERA events triggered by airplane pulses, we first apply the beacon timing calibration on an event-by-event basis, followed by the airplane timing offset analysis. The result is depicted in Fig. 13. We can deduce several results from this graph.

First of all, comparing this result with the analysis of the same airplane events without application of the beacon correction (Fig. [12), it becomes clear that for stations 25 onwards, the mean timing offsets with respect to the reference station (number 40) indeed become close to zero once the beacon analysis is applied. This means that the beacon correction does indeed correctly compensate for GPS clock offsets present in the individual AERA stations.

For stations 1-24, however, there appears to be a systematic time offset of $\approx-65$ ns with respect to the reference station. The main difference between stations 1-24 and stations 25 onwards is the choice of antenna type [20]. For stations 1-24 an LPDA is used, whereas for stations 25 onwards we use a butterfly antenna. The two types of antennas have a different frequency-dependent group delay, which is not taken into account for the beacon correction as applied for this figure.

In principle, the different group delay can be corrected for in the data analysis on the basis of 4NEC2 simulations of the antenna characteristics [19,20]. However, in the latter paper a discrepancy between the measured and simulated group delays is reported, in particular close to the horizon, i.e., for large zenith angles. This discrepancy is the likely cause of the shift between stations with different antenna types visible in Fig. 12, since there the simulated group delay has been applied in the analysis. Thus, the result confirms that the delays predicted by our antenna models probably do not correctly describe the time offsets, which can be due to a problem in the modeling of either the LPDA, the butterfly antenna or both of them.

While the mean time offsets are consistent among stations with the same type of antenna, there is very significant scatter of the offsets determined from individual airplane events around this mean 


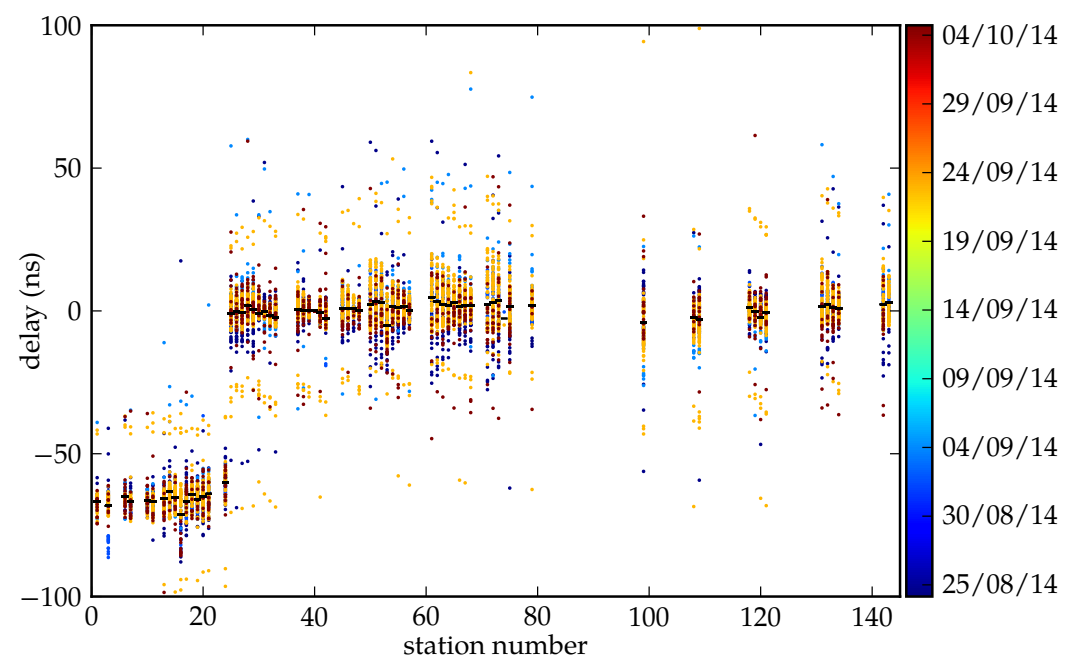

Figure 14. Same as Fig. 13 when applying the combined analysis to all airplanes detected in at least 10 AERA events.

offset. The scatter is obviously not random but shows two important characteristics. First, there is again a seemingly time-ordered distribution with moderate scatter (to be discussed in section 5). Secondly, there are outliers with large time offsets beyond an obvious gap in the distribution. The probable reason for the outliers is that some step in the airplane analysis failed. It can happen, for example, that a neighboring peak in the enveloped airplane pulse is accidentally misidentified as the main peak (cf. Fig. \), especially due to the influence of noise. This leads to a time offset of multiples of $\approx 30 \mathrm{~ns}$ and can likely explain the presence of the outliers with large offsets beyond the gap. We define a cut criterion to remove these outliers: time offsets corresponding to more than \pm 4 MAD (median absolute deviation) from the mean offset of a particular station are cut away.

\subsection{Multiple airplanes}

The analysis of one airplane showed that indeed the beacon timing is able to determine offsets arising from GPS clock drifts on an event-by-event basis. However, so far we only showed this on a short time scale of minutes. The important question is now whether analyses carried out on other detected airplanes on other days and even in other months lead to the same consistent time offsets of the AERA stations.

We apply the same combined beacon and airplane analysis as in the previous subsection to all airplanes that have been detected in at least 10 AERA events. Station 40 is chosen as the reference station in all cases. This results in the data shown in Fig. 14.

It becomes clear that indeed the time offsets for AERA stations 1-24 are consistently established with a mean of $\approx-65 \mathrm{~ns}$ and the time offsets of the stations with butterfly antennas are consistently very close to zero with respect to the reference station 40 . The fact that these results have been achieved from many different airplanes over the course of months illustrates that both methods give consistent results over this long time scale and that indeed the beacon timing method is able to correct for true drifts arising in the GPS clocks of the AERA stations.

We again cut away outliers in the resulting time offsets of more than \pm 4 MAD for each individual airplane separately and show more quantitative results for the resulting distributions in 
Fig. 15. The sub-histograms with different colors show the resulting distributions for different airplanes. The mean time offsets are again consistent with the results presented for the one airplane in the previous subsection.
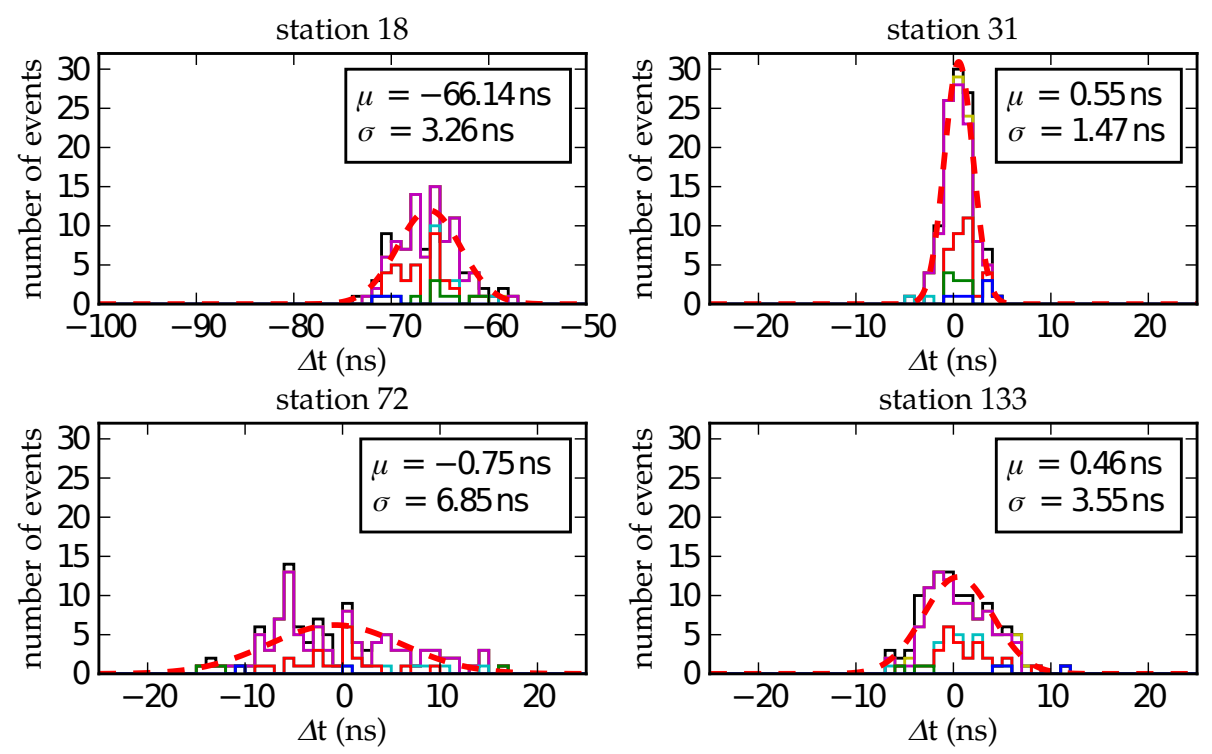

Figure 15. Time offsets determined from events of all detected airplanes in AERA stations 18 (LPDA antenna), 31, 72 and 133 (butterfly antennas). The mean time offsets $\mu$ and their standard deviations $\sigma$ have been calculated after the MAD cut explained in section 4.1 .

A closer look at the distributions determined for individual AERA stations reveals that the widths of the resulting time-offset distributions are not constant but show a correlation with the distance of the chosen AERA station with respect to the reference station 40, see Fig. 16. This effect is expected: the larger the "baseline" over which the time offsets have to be determined, the more precisely the position of the airplane needs to be known. A more sophisticated analysis which does not rely on one particular station as the reference station but uses different reference stations for different parts of the detector array could possibly improve the results, at least lead to a more homogeneous width of the distributions among all AERA stations. We are, however, mostly interested in the means of the distributions, which are already determined well enough for our purposes with the current analysis. This is illustrated in Fig. 17, which demonstrates that the mean time offsets determined over the course of several months from multiple airplane fly-overs are consistent within $\approx 2$ ns among AERA stations using the same antenna type. The small number of outliers visible in Figs. 16 and 177 should be investigated in some more detail once significantly more statistics are available.

\subsection{Reconstruction improvement}

As an example for the improvement in cosmic-ray data analysis achieved with the time calibration, we demonstrate the effect on the reconstruction of the radio wavefront of a cosmic-ray event. The wavefront of radio emission from cosmic-ray showers is known to be of hyperbolic shape $[8,10]$. We analyze an AERA event detected simultaneously with butterfly antennas and LPDAs and fit a 


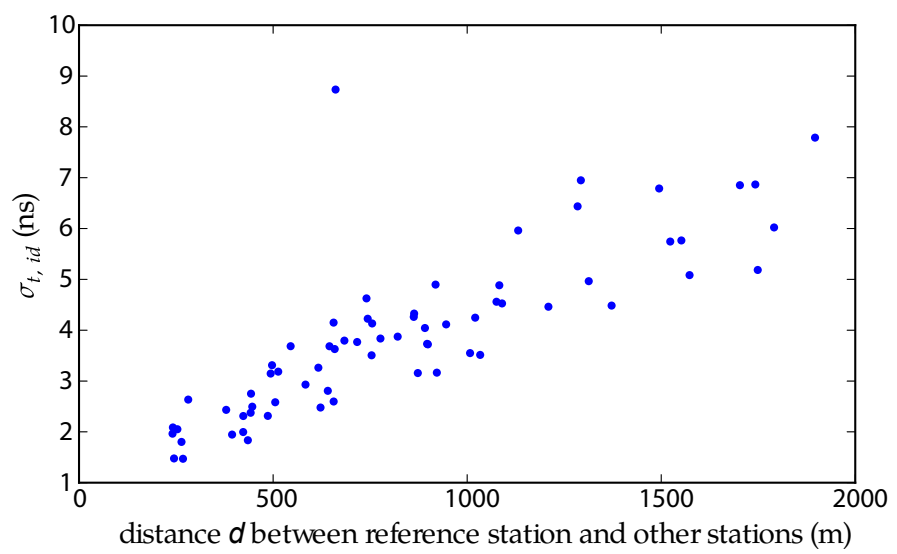

Figure 16. Standard deviation $\sigma_{t, \text { id }}$ as a function of the distance $d$ between the reference station 40 and each individual AERA station id. Each point marks a station.

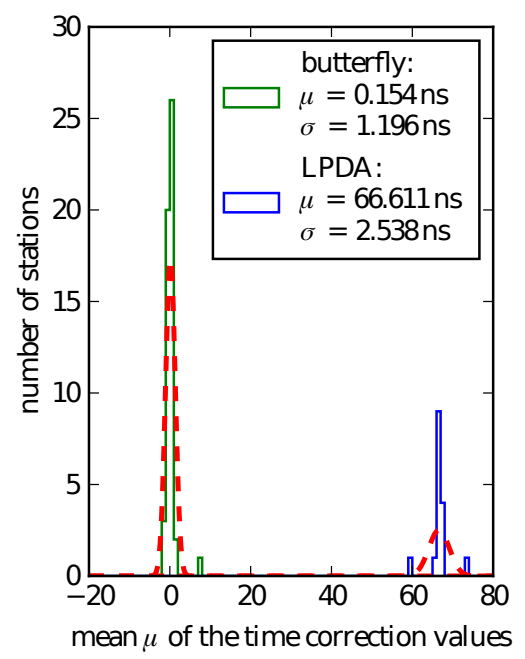

Figure 17. Histogram of the mean $\mu$ of the time correction values. The standard deviations $\sigma$ of the distributions provide a measure for the average agreement between the beacon and airplane methods. The values stated in the statistics box are from fitted Gaussians (dashed lines).

hyperbolic wavefront of the form

$$
t=\beta\left(\sqrt{\left(1+x^{2} / \gamma^{2}\right)}-1\right)
$$

to the measured cosmic-ray data. $\beta$ and $\gamma$ are fit parameters of the hyperbola, $t$ denotes the arrival time of the radio pulse with respect to the arrival time expected for a plane wave, and $x$ denotes the distance of the radio detector station from the air-shower axis. The air-shower axis is determined by the arrival direction and the core position ("impact point") of the extensive air shower. The arrival direction is well-established from the Auger surface detector reconstruction featuring an angular resolution of better than $1^{\circ}$ [13]. Thus, the arrival direction is fixed in the wavefront fit, but the core position reconstructed with the surface detector has significant uncertainties. Hence, the core position is optimized as part of the hyperbolic wavefront fit procedure. 
In Fig. 18 we show a comparison between a wavefront fit without application of the time calibration (left) and with application of the time calibration (right). The improvement in the wavefront fit due to the time calibration is obvious. Even more importantly, however, the fit result with time calibration yields a core position well within the $1 \sigma$ error ellipse of the core position determined with the surface-detector reconstruction. The core position determined without time calibration, in contrast is incompatible with the surface-detector core position (it lies outside the $4 \sigma$ error ellipse). This example clearly illustrates the success and importance of the time calibration using the beacon transmitter and airplanes.
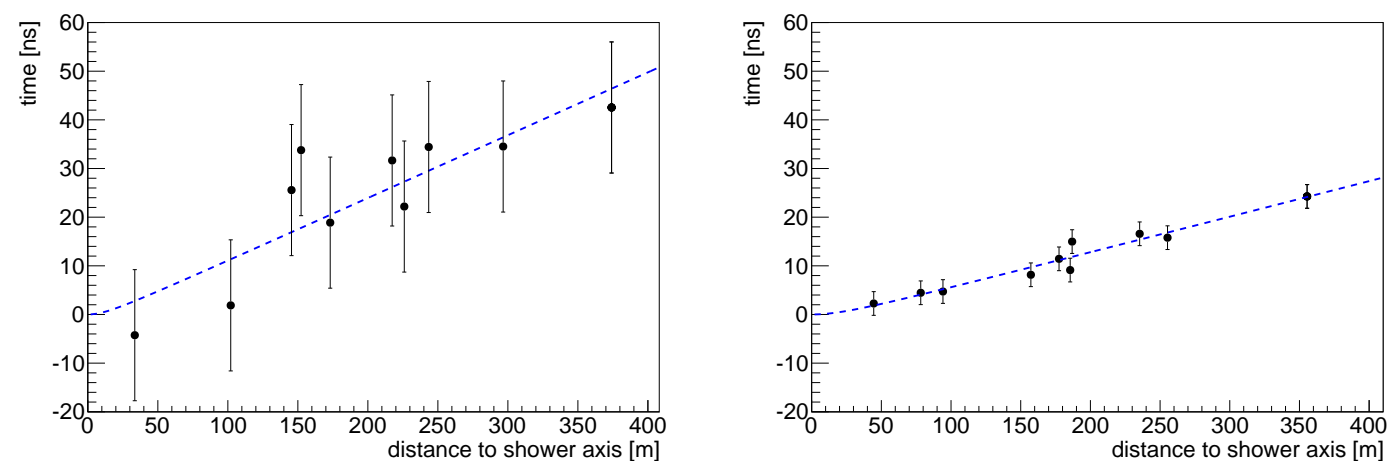

Figure 18. Improvement achieved in the reconstruction of the hyperbolic radio wavefront for a cosmic-ray event measured simultaneously with LPDAs and butterfly antennas of AERA. The left panel shows the result of a hyperbolic wavefront fit without application of the time calibration. The seemingly valid fit yields a core position incompatible with the one reconstructed by the Auger surface detector. The right panel shows the fit result once the time calibration is applied (in both panels uncertainty bars are the timing uncertainty, before and after applying the beacon method, respectively). The hyperbolic wavefront fits the measured pulse arrival times well and the resulting core position is in good agreement with the surface-detector reconstruction.

\section{Discussion}

The analysis presented here showed conclusively that both the beacon-timing calibration method and the airplane-timing calibration method give consistent results. With this we have reached our main goal, to demonstrate that the beacon-timing calibration can continuously correct for GPS clock drifts. Also, we have established a systematic time offset between stations with LPDA and butterfly antennas that is not correctly accounted for by our current antenna models. A detailed verification of our antenna models for all possible directions, however, is very difficult with this approach as only selected arrival directions are available in the measurement. Activities are thus ongoing within AERA to cross-check the antenna models with dedicated calibration campaigns employing reference sources on an octocopter. This will be important especially for air-shower events recorded simultaneously with the two different antenna types, since model uncertainties cancel out for those events recorded only in stations with a unique antenna type.

The airplane-timing calibration is in principle not needed anymore in the future, as the beacontiming correction has been shown to work reliably. For this reason we did not invest further work to improve the airplane analysis. There are a number of improvements that could still be made. For 
example, the refractive index of the atmosphere is currently set to a constant value, resulting in a typical delay of a few ns. A height-dependent refractive index gradient can be applied in the analysis for increased precision. Tests with different choices for the refractive index, however, revealed that this improvement will be small compared to the size of the systematic effects observed.

As mentioned before, the choice of a fixed reference station for the whole analysis leads to unevenly distributed systematic uncertainties, which increase with the distance from the reference station. An analysis strategy using different reference stations for different parts of the array could distribute these uncertainties in a more homogeneous fashion.

The altitude of the airplanes reported in the ADS-B packets is the barometric height, which we interpreted as altitude above ground in our analysis, but in principle could be corrected for atmospheric conditions at the time of flight. This could yield a more precise airplane location and thus improved accuracy of the analysis. Likewise, interpolation of the airplane position between ADS-B data points could be improved, trying to take into account changes in heading and altitude occurring over the course of the trajectory.

The most important issue that should be investigated further is the origin of the time ordering observed in the event-by-event time offsets determined with the airplane calibration. These indicate some direction dependence of the delays, which is difficult to study in detail, since the recorded airplanes cover a limited range in zenith and azimuth, only (cf. Fig. Q). This time-ordering effect could possibly be related to a systematic offset between the reported ADS-B position and the position of the radio pulse transmitter onboard the aircraft. The fact that the mean values determined from the distributions are in such good agreement among detector stations with the same type of antenna gives us confidence that the mean values are unbiased. Nevertheless, this effect should be studied further, in particular when data with larger statistics and from more directions are available.

Assuming that the uncertainty of the ADS-B position is dominated by the difference between barometric and geometric height, we estimated the size of the effect on the basis of pressure variations in the atmospheric models used for the Auger fluorescence detectors. Indeed the altitude difference can be on the order of several hundred meters in extreme cases. A change of altitude of this order is sufficient to change the results for individual airplanes, and can reduce systematic effects, e.g., the time ordering or the distance effect shown in Fig. 16. However, we do not know the true airplane position. Also, analyses with varying altitudes revealed that an altitude correction cannot explain all effects simultaneously, and the average agreement between beacon and airplane analysis remains at the level of $2 \mathrm{~ns}$. Consequently, the effect of the uncertainty in the airplane position has to be studied in more detail, and the dominant uncertainty in the altitude cannot be the only explanation for the systematic uncertainties of the airplane method.

An attractive option for the future could be to perform a targeted search for radio pulses from the International Space Station or commercial satellites. If these do emit radio pulses in our frequency band, they can be used as a regular source for timing calibration. As these sources are at larger distances on well-defined orbits, the achievable precision should be better than for airplanes, and also observation over a wider range of zenith and azimuth angles would be possible.

Finally, it is worth investigating the potential of using airplane signals also for other purposes, in particular relative amplitude calibration and antenna directivity studies of radio detector stations. 


\section{Conclusions}

The Auger Engineering Radio Array needs a relative timing accuracy on the order of a nanosecond to enable interferometric and wavefront analyses of the radio emission from extensive air showers. GPS timing alone cannot provide sufficient timing accuracy as there are significant drifts occurring in the GPS clocks. To achieve the desired timing in this distributed detector only connected via wireless communications, we employ two methods for precise time calibration. The AERA reference beacon transmits four continuous sine wave signals which are recorded in our physics data. From the relative phases of the received sine waves, GPS clock drifts can be corrected. Up to now it was unclear whether the drifts seen by the beacon represent only intrinsic GPS clock drifts or whether they could also be influenced by propagation and environmental effects. Therefore, we have cross-checked the beacon method with an independent time-calibration technique. We can now exclude that the observed drifts are an artifact of the beacon analysis, since the calibration with airplane signals from different directions yields consistent results.

A low-cost software-defined radio solution continuously monitors the vicinity of AERA for ADS-B signals transmitted by commercial airplanes. These signals contain real-time position information about airplanes at a distance of hundreds of $\mathrm{km}$. Some airplanes also emit pulsed radio signals in the frequency range of AERA (30-80 MHz). If an airplane approaches AERA, the central data acquisition is notified to let triggers from pulsed airplane signals pass through. An offline analysis using the position information from the ADS-B data and the radio pulses recorded in the AERA data is then able to establish the mean time offsets between AERA stations. A combined analysis with the beacon-timing correction shows that both are consistent within $\approx 2$ ns. Consequently each individual method is accurate to at least $2 \mathrm{~ns}$. One of the methods, i.e., either the beacon or the airplane method, could well have a better accuracy than this since the comparison then would be dominated by the less accurate method. In addition to this result, a previously unknown offset of several times 10 ns between AERA stations with LPDA and butterfly antennas was established with the airplane calibration.

The analysis shown here establishes that the beacon-timing calibration works as intended and can be used for event-by-event time calibration of all data acquired with AERA. The accuracy is of $\approx 2 \mathrm{~ns}$ or better, and future tests will show if the desired accuracy of $1 \mathrm{~ns}$ is achieved already.

\section{Acknowledgments}

We would like to thank several people who supported this work directly or by their involvement in preparatory studies: H. Bolz, H. Bozdog, D. Huber, M. Konzack, R. Rink, F. Leven. We are also grateful to the anonymous referee for useful suggestions and comments.

The successful installation, commissioning, and operation of the Pierre Auger Observatory would not have been possible without the strong commitment and effort from the technical and administrative staff in Malargüe. We are very grateful to the following agencies and organizations for financial support:

Comisión Nacional de Energía Atómica, Agencia Nacional de Promoción Científica y Tecnológica (ANPCyT), Consejo Nacional de Investigaciones Científicas y Técnicas (CONICET), Gobierno de la Provincia de Mendoza, Municipalidad de Malargüe, NDM Holdings and Valle Las 
Leñas, in gratitude for their continuing cooperation over land access, Argentina; the Australian Research Council (DP150101622); Conselho Nacional de Desenvolvimento Científico e Tecnológico (CNPq), Financiadora de Estudos e Projetos (FINEP), Fundação de Amparo à Pesquisa do Estado de Rio de Janeiro (FAPERJ), São Paulo Research Foundation (FAPESP) Grants No. 2010/07359-6 and No. 1999/05404-3, Ministério de Ciência e Tecnologia (MCT), Brazil; Grant No. MSMT-CR LG13007, No. 7AMB14AR005, and the Czech Science Foundation Grant No. 14-17501S, Czech Republic; Centre de Calcul IN2P3/CNRS, Centre National de la Recherche Scientifique (CNRS), Conseil Régional Ile-de-France, Département Physique Nucléaire et Corpusculaire (PNC-IN2P3/CNRS), Département Sciences de l'Univers (SDU-INSU/CNRS), Institut Lagrange de Paris (ILP) Grant No. LABEX ANR-10-LABX-63, within the Investissements d'Avenir Programme Grant No. ANR-11-IDEX-0004-02, France; Bundesministerium für Bildung und Forschung (BMBF), Deutsche Forschungsgemeinschaft (DFG), Finanzministerium BadenWürttemberg, Helmholtz Alliance for Astroparticle Physics (HAP), Helmholtz-Gemeinschaft Deutscher Forschungszentren (HGF), Ministerium für Wissenschaft und Forschung, Nordrhein Westfalen, Ministerium für Wissenschaft, Forschung und Kunst, Baden-Württemberg, Germany; Istituto Nazionale di Fisica Nucleare (INFN), Istituto Nazionale di Astrofisica (INAF), Ministero dell'Istruzione, dell'Universitá e della Ricerca (MIUR), Gran Sasso Center for Astroparticle Physics (CFA), CETEMPS Center of Excellence, Ministero degli Affari Esteri (MAE), Italy; Consejo Nacional de Ciencia y Tecnología (CONACYT), Mexico; Ministerie van Onderwijs, Cultuur en Wetenschap, Nederlandse Organisatie voor Wetenschappelijk Onderzoek (NWO), Stichting voor Fundamenteel Onderzoek der Materie (FOM), Netherlands; National Centre for Research and Development, Grants No. ERA-NET-ASPERA/01/11 and No. ERA-NET-ASPERA/02/11, National Science Centre, Grants No. 2013/08/M/ST9/00322, No. 2013/08/M/ST9/00728 and No. HARMONIA 5 - 2013/10/M/ST9/00062, Poland; Portuguese national funds and FEDER funds within Programa Operacional Factores de Competitividade through Fundação para a Ciência e a Tecnologia (COMPETE), Portugal; Romanian Authority for Scientific Research ANCS, CNDI-UEFISCDI partnership projects Grants No. 20/2012 and No. 194/2012, Grants No. 1/ASPERA2/2012 ERA-NET, No. PN-II-RU-PD-2011-3-0145-17 and No. PN-II-RU-PD-2011-3-0062, the Minister of National Education, Programme Space Technology and Advanced Research (STAR), Grant No. 83/2013, Romania; Slovenian Research Agency, Slovenia; Comunidad de Madrid, FEDER funds, Ministerio de Educación y Ciencia, Xunta de Galicia, European Community 7th Framework Program, Grant No. FP7-PEOPLE-2012-IEF-328826, Spain; Science and Technology Facilities Council, United Kingdom; Department of Energy, Contracts No. DE-AC0207CH11359, No. DE-FR02-04ER41300, No. DE-FG02-99ER41107 and No. DE-SC0011689, National Science Foundation, Grant No. 0450696, The Grainger Foundation, USA; NAFOSTED, Vietnam; Marie Curie-IRSES/EPLANET, European Particle Physics Latin American Network, European Union 7th Framework Program, Grant No. PIRSES-2009-GA-246806; and UNESCO. 


\section{Appendix: Detected Airplanes}

Table 1. Airplanes detected with AERA in the time from June 20 to October 5, 2014. Only detections fulfilling the following quality cuts are listed: detection in at least 10 detector stations with a signal-to-noise ratio of at least 15 and a reconstructed radius of curvature of the spherical wavefront between $7.5 \mathrm{~km}$ and $99 \mathrm{~km}$ as well as a reconstructed zenith angle less than $80^{\circ}$. We do not know, why the number of events varies strongly, i.e., why a few airplanes emit significantly more detectable pulses than others.

\begin{tabular}{ccccc}
\hline UTC date & events & Mode-S call sign & airplane type & airline \\
\hline 20/06/14, 16:26 & 3 & C05843 & Boeing 767-35H & Air Canada \\
22/07/14, 03:26 & 1 & E48987 & Boeing 737-8EH & GOL Transportes \\
22/07/14, 03:34 & 6 & E47FE0 & Airbus A320-232 & TAM Linhas Aereas \\
28/07/14, 15:43 & 1 & E06541 & Boeing 737-81D & Aerolineas Argentinas \\
31/07/14, 06:29 & 1 & E80320 & Airbus A319-111 & Sky Airline \\
31/07/14, 11:28 & 4 & E48C03 & Boeing 737-8HX & GOL Transportes \\
31/07/14, 18:20 & 1 & E48987 & Boeing 737-8EH & GOL Transportes \\
03/08/14, 11:27 & 1 & E48986 & Boeing 737-8EH & GOL Transportes \\
24/08/14, 03:36 & 16 & E48854 & Boeing 737-8EH & GOL Transportes \\
24/08/14, 11:30 & 17 & E48854 & Boeing 737-8EH & GOL Transportes \\
01/09/14, 11:23 & 32 & E48854 & Boeing 737-8EH & GOL Transportes \\
03/09/14, 21:21 & 11 & E48C04 & Boeing 737-8HX & GOL Transportes \\
08/09/14, 21:33 & 9 & E48C03 & Boeing 737-8HX & GOL Transportes \\
08/09/14, 22:07 & 4 & E80411 & Airbus A320-233 & LAN Airlines \\
10/09/14, 01:58 & 1 & E80413 & Airbus A320-233 & LAN Airlines \\
13/09/14, 13:02 & 3 & E80414 & Airbus A320-233 & LAN Airlines \\
22/09/14, 21:16 & 115 & E48854 & Boeing 737-8EH & GOL Transportes \\
28/09/14, 21:27 & 1 & E48C04 & Boeing 737-8HX & GOL Transportes \\
02/10/14, 14:27 & 1 & $0 C 208 E$ & Boeing 737-8V3 & Copa Airlines \\
04/10/14, 11:28 & 14 & E48985 & Boeing 737-8EH & GOL Transportes \\
04/10/14, 17:13 & 33 & E48853 & Boeing 737-8EH & GOL Transportes \\
\hline
\end{tabular}

\section{References}

[1] T. Huege, The Renaissance of Radio Detection of Cosmic Rays, Braz. J. Phys. 44 (2014) 520.

[2] LOPES Collaboration, W.D. Apel, et al., Reconstruction of the energy and depth of maximum of cosmic-ray air showers from LOPES radio measurements, Phys. Rev. D 90 (2014) 062001.

[3] Pierre Auger Collaboration, A. Aab, et al., The energy in the radio signal of extensive air showers, Phys. Rev. Lett. submitted (2015), and

Pierre Auger Collaboration, A. Aab, et al., Energy Estimation of Cosmic Rays with the Engineering Radio Array of the Pierre Auger Observatory, Phys. Rev. D submitted (2015), arXiv:1508.04267. 
[4] F.G. Schröder for the Pierre Auger Collaboration, Radio detection of high-energy cosmic rays with the Auger Engineering Radio Array, Nucl. Instrum. Meth. A in press (2015), doi:10.1016/j.nima.2015.08.047.

[5] LOFAR Collaboration, S. Buitink, et al., Method for high precision reconstruction of air shower $X_{\max }$ using two-dimensional radio intensity profiles, Phys. Rev. D 90 (2014) 082003.

[6] Tunka-Rex Collaboration, P.A. Bezyazeekov, et al., Radio measurements of the energy and depth of maximum of cosmic-ray air showers by Tunka-Rex, J. Cosmol. Astropart. P. 01 (2016) 052.

[7] LOPES Collaboration, H. Falcke, et al., Detection and imaging of atmospheric radio flashes from cosmic ray air showers, Nature 435 (2005) 313.

[8] LOPES Collaboration, W.D. Apel, et al., The wavefront of the radio signal emitted by cosmic ray air showers, J. Cosmol. Astropart. P. 09 (2014) 025.

[9] F.G. Schröder, T. Asch, L. Bähren, et al., New method for the time calibration of an interferometric radio antenna array, Nucl. Instr. Meth. A 615 (2010) 277.

[10] LOFAR Collaboration, A. Corstanje, et al., The shape of the radio wavefront of extensive air showers as measured with LOFAR, Astropart. Phys. 61 (2015) 22.

[11] J. Serrano, P. Alvarez, M. Cattin, et al., The White Rabbit Project, in proceedings of ICALEPCS TUC004, 2009, Kobe, Japan.

[12] T. Huege for the Pierre Auger Collaboration, Radio detection of cosmic rays in the Pierre Auger Observatory, Nucl. Instrum. Meth. A 617 (2009) 484.

[13] Pierre Auger Collaboration, A. Aab, et al., The Pierre Auger Cosmic Ray Observatory, Nucl. Instrum. Meth. A 798 (2015), 172.

[14] i-Lotus Corporation Pte. Ltd. Singapore, M12M Timing Oncore ${ }^{\mathrm{TM}}$ Receiver, Technical Data Sheet Rev. 1.1, 2008.

[15] B. Revenu for the Pierre Auger Collaboration, Autonomous detection and analysis of radio emission from air showers at the Pierre Auger Observatory, in Proceedings of 32nd ICRC, 2011, Beijing, China, 3 (2011) 176; arXiv:1107.4807.

[16] Pierre Auger Collaboration, P. Abreu, et al, S. Acounis, D. Charrier, T. Garçon, C. Rivière, P. Stassi, Results of a self-triggered prototype system for radio-detection of extensive air showers at the Pierre Auger Observatory, J. Instrum. 7 (2012) P11023.

[17] Federal Aviation Administration, Automatic Dependent Surveillance Broadcast (ADS-B) Out Performance Requirements to Support Air Traffic Control (ATC) Service, Federal Register 75 (2010) 103.

[18] Private communication with Airbus and the DGAC ("Direction Générale de l'aviation civile", the French national authority for civilian aviation).

[19] Pierre Auger Collaboration, P. Abreu, et al., Advanced functionality for radio analysis in the Offline software framework of the Pierre Auger Observatory, Nucl. Instrum. Meth. A 635 (2011) 92.

[20] Pierre Auger Collaboration, P. Abreu, et al., Antennas for the detection of radio emission pulses from cosmic-ray induced air showers at the Pierre Auger Observatory, J. Instrum. 7 (2012) P10011. 